\title{
Analytical solutions of ac electrokinetics in interdigitated electrode arrays: Electric field, dielectrophoretic and traveling-wave dielectrophoretic forces
}

\author{
Tao Sun, ${ }^{*}$ Hywel Morgan, and Nicolas G. Green ${ }^{\dagger}$ \\ Nanoscale Systems Integration Group, School of Electronics and Computer Science, University of Southampton, \\ SO17 1BJ United Kingdom
}

(Received 5 June 2007; revised manuscript received 21 August 2007; published 25 October 2007)

\begin{abstract}
Analysis of the movement of particles in a nonuniform field requires accurate knowledge of the electric field distribution in the system. This paper describes a method for analytically solving the electric field distribution above interdigitated electrode arrays used for dielectrophoresis (DEP) and traveling wave dielectrophoresis (twDEP), using the Schwarz-Christoffel mapping method. The electric field solutions are used to calculate the dielectrophoretic force in both cases, and the traveling wave dielectrophoretic force and the electrorotational torque for the twDEP case. This method requires no approximations and can take into account the Neumann boundary condition used to represent an insulating lid and lower substrate. The analytical results of the electric field distributions are validated for different geometries by comparison with numerical simulations using the finite element method.
\end{abstract}

DOI: 10.1103/PhysRevE.76.046610

PACS number(s): 41.20.- $-\mathrm{q}$

\section{INTRODUCTION}

In the field of micro total analysis systems ( $\mu \mathrm{TAS})$ or the lab-on-a-chip (LOC), the manipulation, separation, and characterization of biological particles can be performed using electrical techniques based on ac electrokinetics [1]. When an electric field is applied to a suspension of particles, charges accumulate at the interface between the particle and the fluid medium, a phenomenon known as Maxwell-Wagner interfacial polarization. This produces an induced dipole moment across the particle, the magnitude and direction depending on the difference in the polarizability of the particle and the medium. The interaction between the induced dipole moment and a nonuniform electric field produces an unbalanced force on the two poles, which results in the translational movement of the particles. Applications in this area include manipulating, separating, trapping and sorting particles such as cells, bacteria, and viruses [2-8], using dielectrophoresis (DEP) [9,10], electrorotation (ROT) $[11,12]$, and traveling wave dielectrophoresis (twDEP) [13-15].

DEP is the movement of the particles in the nonuniform electric field, due to the imbalance of force on the two sides (poles) of the particle. This movement is towards regions of high or low field strength depending on whether the particle is more or less polarizable than the medium at the applied frequency. The DEP force depends on the in-phase component of the effective dipole [16,17]. ROT occurs when the field has a nonuniform phase, which means that there is a rotational component to the field. The direction of the effective dipole lags behind the turning field vector, leading to torque on the particle which depends on the out-of-phase component of the dipole $[18,19]$. For further details on the relationship between DEP and ROT see Wang et al. [20,21]. In electric fields with spatially varying phases, a particle ex-

\footnotetext{
*ts04r@ecs.soton.ac.uk

†ng2@ecs.soton.ac.uk
}

periences a linear force in addition to the DEP force which depends on the out-of-phase component of the effective dipole. This is referred to as traveling wave dielectrophoresis (twDEP), since it was first observed in traveling electric fields [22]. This method can be used to separate or fractionate particles without the need to pump a liquid through the device.

A number of microfluidic devices with different microchannel geometries and microelectrode patterns have been used for DEP and twDEP. Since the interaction between the electric field and the induced dipole is the origin of the motion of the particles, it is important to characterize the strength and direction of the electric field in the system. Unfortunately, accurate analytical electric field solutions are generally difficult to obtain due to the complicated structures of the microelectrodes and the geometries of the microdevices. Even for the most commonly used interdigitated electrode arrays, no strict and computationally efficient analytical solution has been reported. Previous analytical solutions use Green's theorem [23], Green's function [24,25], halfplane Green's function [26] and Fourier series [27,28]. However, these analytical solutions all involve approximations. In both the Green's theorem and Fourier series methods, it is assumed that the potential varies linearly with distance in the electrode gaps. In the method of Green's function, the gradient of the electric field magnitude squared is influenced by the choice of a characteristic length scale. In the method of half-plane Green's function, a linear approximation for the surface potential in the gaps between the electrodes is adopted. Other approaches, such as the charge density method [29] and numerical simulation [30] are accurate but computationally expensive.

In this paper, we present analytical solutions of the electric field distribution above interdigitated electrode arrays in both DEP and twDEP applications using the SchwarzChristoffel mapping (SCM) method. This method requires no approximations and the results are straightforward to use. In other analytical solutions [23-27], the presence of the upper 


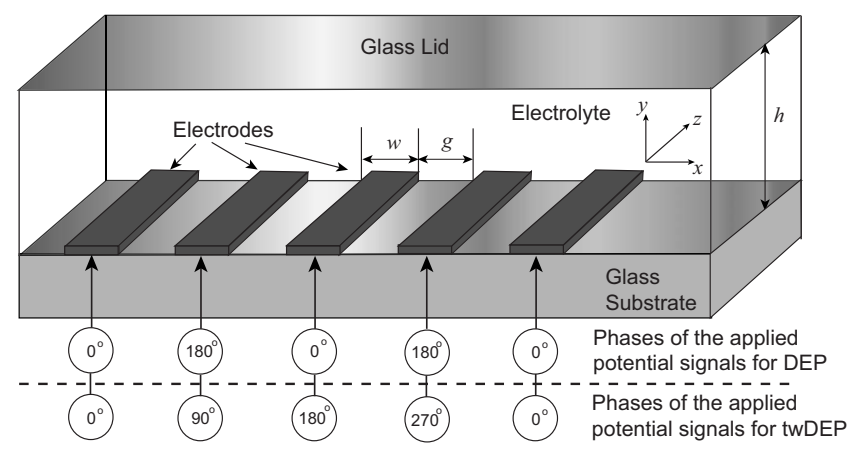

FIG. 1. Diagram showing the experimental system, consisting of the interdigitated electrode array used for dielectrophoresis (DEP) and traveling wave dielectrophoresis (twDEP). For DEP, the electrodes are connected to voltages with $180^{\circ}$ phase shifts. For twDEP, the electrodes are connected to a frequency generator with $90^{\circ}$ phase shift. $w$ is the electrode width, $g$ is the electrode gap, and $h$ is the height of the channel.

surface of the fluidic channel (the insulating lid in real devices) imposes a Neumann condition on the solution of the potential, a factor that was not considered. In Ref. [28], this condition was analyzed using a closed form of Fourier series, but the solution approximates the potential distribution in the electrode gaps to a linear function.

When interdigitated electrode arrays are used for the application of DEP, two ac voltage signals of angular frequency $\omega$ with phases $0^{\circ}$ and $180^{\circ}$ are alternately connected to the electrodes to generate a nonuniform electric field with a spatially constant phase. For twDEP, four signals with phase shifts of $90^{\circ}$ are sequentially applied to the electrodes in the array, generating a traveling electric field with a spatially dependent phase. Figure 1 shows a schematic of the system and the geometrical parameters for the electrodes and the channel with the different potentials for DEP and twDEP, respectively.

In Sec. II, we briefly review fundamental ac electrokinetic theory and the principle of SCM method with the common boundary conditions used in the electric field analysis of DEP and twDEP electrode arrays. Due to the different applied potentials in DEP and twDEP electrode arrays, the geometries of the problem spaces are different and the solutions for the two cases are discussed in Secs. III and IV separately. Analytical electric field solutions are derived in both cases and validated by numerical simulations using the finite element method (FEM). The field solutions are used to calculate the DEP force in the DEP and twDEP arrays and twDEP force and ROT torque in the twDEP array.

\section{THEORY}

For DEP and twDEP experiments and calculations, the general approach is to assume that the field is quasielectrostatic $[1,24,27,30]$. This assumes that the electric field is irrotational (an implicit assumption in the derivation of the DEP and twDEP forces), or that the currents in the system are slow time-varying. As a result, the electric field can be taken to be the gradient of a scalar potential.

\section{A. Fields and DEP force in interdigitated electrode arrays}

For an ac potential of single frequency, the electric field can be expressed using complex phasor notation $[1,11,14]$ as $\mathbf{E}=\operatorname{Re}\left[\widetilde{\mathbf{E}} e^{j \omega t}\right]$, where $j^{2}=-1, \omega$ is the angular frequency of the electric field, $t$ is time, $\operatorname{Re}[\cdots]$ indicates the real part of $[\cdots]$ and $\widetilde{\mathbf{E}}=-\boldsymbol{\nabla} \widetilde{\phi}=-\left(\boldsymbol{\nabla} \phi_{R}+j \boldsymbol{\nabla} \phi_{I}\right)$ is the general complex amplitude of the electric field. $\widetilde{\phi}=\phi_{R}+j \phi_{I}$ is the complex electrical potential phasor with $\phi_{R}$ and $\phi_{I}$ as the real and imaginary components respectively. [1]

The time-averaged dielectrophoretic force on the dipole is

$$
\langle\mathbf{F}\rangle=\frac{1}{2} \operatorname{Re}\left[(\widetilde{\mathbf{p}} \cdot \boldsymbol{\nabla}) \widetilde{\mathbf{E}}^{*}\right]=\frac{1}{2} v \operatorname{Re}\left[\widetilde{\alpha}(\widetilde{\mathbf{E}} \cdot \nabla) \widetilde{\mathbf{E}}^{*}\right],
$$

where $\widetilde{\mathbf{p}}$ is the induced dipole moment phasor, $v$ the volume of the particle, $\widetilde{\alpha}$ the effective polarizability, and $*$ indicates complex conjugate.

Equation (1) can be rewritten as

$$
\langle\mathbf{F}\rangle=\frac{1}{4} v \operatorname{Re}[\widetilde{\alpha}] \nabla|\widetilde{\mathbf{E}}|^{2}-\frac{1}{2} v \operatorname{Im}[\widetilde{\alpha}](\boldsymbol{\nabla} \times(\operatorname{Re}[\widetilde{\mathbf{E}}] \times \operatorname{Im}[\widetilde{\mathbf{E}}])),
$$

where $|\widetilde{\mathbf{E}}|^{2}=|\operatorname{Re}[\widetilde{\mathbf{E}}]|^{2}+|\operatorname{Im}[\widetilde{\mathbf{E}}]|^{2}$ and $\operatorname{Im}[\cdots]$ indicates the imaginary part of $[\cdots]$.

Equation (2) shows that the dielectrophoretic force consists of two components; the first term on the right is the conventional DEP force; the second term the twDEP force. Conventional DEP occurs in a nonuniform electric field. In order to calculate the DEP force, we need to know the volume and polarizability of the particle and the field-related component, $\boldsymbol{\nabla}|\widetilde{\mathbf{E}}|^{2}$, which we term the "dielectrophoretic force component" in this paper, and obtained from field analysis. The twDEP force occurs whenever the imaginary (out-of-phase) part of the effective polarizability is nonzero and giving a spatial phase variation. As particles move in the $x-y$ 2D plane (Fig. 1), they will simultaneously experience an electrorotational torque in the third direction ( $z$ axis). This ROT torque is given by

$$
\langle\Gamma\rangle=\frac{1}{2} \operatorname{Re}\left[\widetilde{\mathbf{p}} \times \widetilde{\mathbf{E}}^{*}\right]=-v \operatorname{Im}[\widetilde{\alpha}](\operatorname{Re}[\widetilde{\mathbf{E}}] \times \operatorname{Im}[\widetilde{\mathbf{E}}]) .
$$

Similarly, the field-related components $\boldsymbol{\nabla} \times(\operatorname{Re}[\widetilde{\mathbf{E}}]$ $\times \operatorname{Im}[\tilde{\mathbf{E}}])$ and $\operatorname{Re}[\tilde{\mathbf{E}}] \times \operatorname{Im}[\tilde{\mathbf{E}}]$ are termed the "traveling wave dielectrophoretic force component" and the "electrorotational torque component," respectively, and are obtained from the analytical electric field solution.

\section{B. Schwarz-Christoffel mapping (SCM) method}

The SCM method maps the upper half of a complex plane ( $T$-plane) into the interior of a given polygon in another complex plane (Z-plane). The $Z$-plane represents the real system in our case. Complex numbers in this plane represent vectors in the real system, with the real component representing the horizontal direction ( $x$-axis) and the imaginary component the vertical direction ( $y$-axis). A general mathematical de- 
scription of the SCM method is provided in Appendix A, and further details can be found in [31].

For the electric field analysis, a nonuniform twodimensional electric field polygonal region is transformed into an equivalent rectangular region (a parallel plate capacitor), where the electric field distribution is uniform, as follows.

(a) Select a basic cell for the physical two-dimensional geometry using symmetrical axes in the physical plane (Z-plane). Determine the boundary conditions (i.e., Neumann or Dirichlet condition) along each boundary of the cell.

(b) Apply SCM method to map the basic cell from the $Z$-plane to the upper half of the auxiliary plane ( $T$-plane).

(c) Apply a second SCM method to transform the upper half of the $T$-plane into a closed parallel plate capacitor region in the model plane ( $W$-plane).

The original nonuniform field problem in the $Z$-plane can then be easily solved in the $W$-plane. The details of the transformation procedure along with the analysis of the electric field are described in the following sections.

The SCM method has been used to solve problems in electrostatics and magnetostatics [32,33], transmission lines and waveguides [34-37], temperature distribution and heat transfer [38,39] and fluid flow [40]. Latterly, conformal mapping has been used to analyze electromagnetic field problems in MEMS devices, including coupling capacitance in combfinger actuators [41] and forces in electromechanical actuators [42]. Gevorgian's group [43-46] has used conformal mapping to model interdigital capacitors (IDC) or coplanarstrip waveguides (CPW), which have similar geometry to the interdigitated electrode arrays. In particular, in Ref. [43], the periodical structure of a single-layer substrate IDC has the same geometry and boundary conditions as the DEP electrode array. However the authors used a metal strip assumption to simplify the problem by changing the closed geometry to a semi-infinite channel, in order to avoid the complex expressions in the transformations for the original geometry, which involve both Jacobian elliptic functions and elliptic integrals. In this paper, we solve the problem of the DEP electrode array without altering the geometry. The derived expressions of the elliptic integrals can be also applied to calculate the capacitances of the IDC in [43] as a complete solution, but this work is beyond the scope of this paper. We are interested in utilizing the analytical electric field solution to characterize the translational motion of the particles in the electrode arrays. For the twDEP electrode array, to the best of our knowledge, there is no accurate analytical solution for four-phase periodic potential.

\section{Common boundary conditions for DEP and twDEP electrode arrays}

Before performing the electric field analysis, the boundary conditions in the corresponding system must be determined. There are several common boundary conditions to both the DEP electrode array and twDEP electrode array.

(a) Since the electrodes are long compared to their width, the problem reduces to two dimensions, allowing the SCM method to be used. (b) The electrodes are much thinner than the electrode width and the gap, so that the electrodes can be represented by a thin section of the bottom boundary at a fixed potential.

(c) Since the normal component of the total current passing through the electrolyte-lid and electrolyte-substrate interfaces must be continuous and the lid and the substrate are made from glass, which has a much smaller permittivity and conductivity than the electrolyte (water) in the channel, the normal component of the electric field in the electrolyte at the interface is negligible compared to that of the glass [30]. Therefore, we assume that Neumann boundary condition (insulating) holds for the potential at the electrolyte-lid and electrolyte-substrate interfaces: $(\partial \widetilde{\phi} / \partial n=0$, where $n$ is the normal to the boundary). The maximum error due to the assumption of Neumann boundary condition at the waterglass interface is found to be less than $1 \%$ of the applied voltage which occurs at the top lid [47].

The remaining boundary conditions in DEP and twDEP electrode arrays depend on the potentials used for each system.

\section{DIELECTROPHORETIC ELECTRODE ARRAY}

The SCM method allows analysis of systems with or without an insulating lid. Therefore in order to demonstrate the effect of the lid on the electric field distribution in the DEP array, we first solve the electric field distribution with complete boundary conditions including an insulating lid. Secondly, the same system is solved without the lid, extending the upper surface to infinity. The resulting analytical solutions of the electric field are compared with numerical simulations. The effect of the lid is discussed for the near and far field regions, equivalent to a shallow and deep channel. The dielectrophoretic force is then calculated from the field solutions and compared with Fourier series analytical solutions.

\section{A. Boundary conditions for array with lid}

The values for the real and imaginary parts of the potential phasor at each electrode, together with the boundary conditions are shown in Fig. 2(a). Since the two ac signals of angular frequency $\omega$ with phases $0^{\circ}$ and $180^{\circ}$ are alternately connected to the electrodes, the boundary conditions are equivalent to two in-phase signals with opposite sign and the imaginary part of the potential phasor is zero $\left(\phi_{I}=0\right)$ and only the real part $\phi_{R}$ needs to be solved. From symmetry, the basic cell $(A B C D E)$ is chosen for the electric field analysis. For a detailed description of the boundary conditions in the DEP array, see [30].

Figure 2(b) shows the three complex planes used for the mapping procedure. The selected cell $A B C D E$ is rotated $90^{\circ}$ and set in the $Z$-plane with the boundary conditions for $\phi_{R}$ as shown: $\partial \phi_{R} / \partial n=0$, along the insulating walls $A E$ and $C D$ and the boundary for the axis of even symmetry $(B C)$. Dirichlet boundary conditions define the fixed potential $\phi_{R}=V$ along the electrode $(A B)$ and the boundary for the axis of odd symmetry, $\phi_{R}=0(D E)$. The complex coordinates for each point in the physical plane (according to the geometrical pa- 


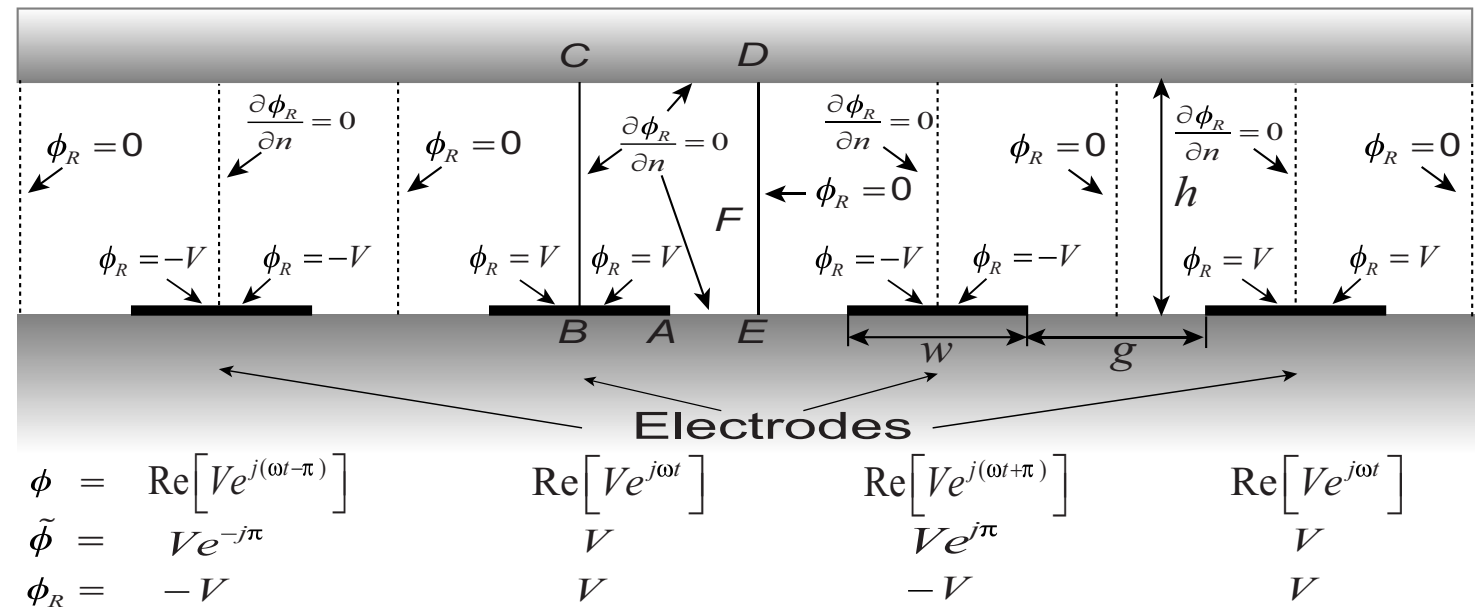

(a)

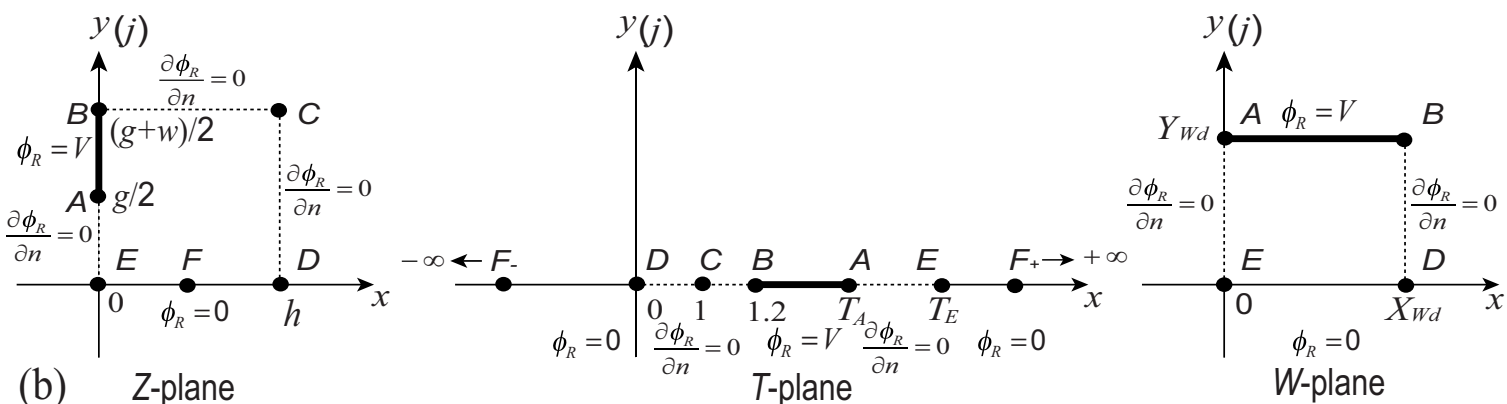

(b)

Z-plane

$T$-plane

W-plane

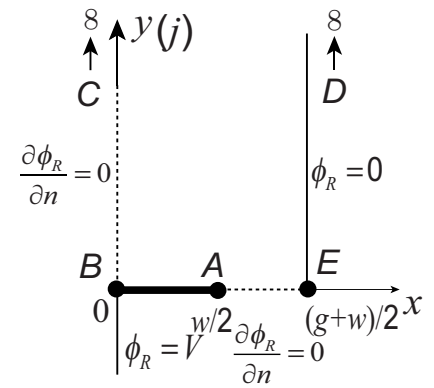

(c)

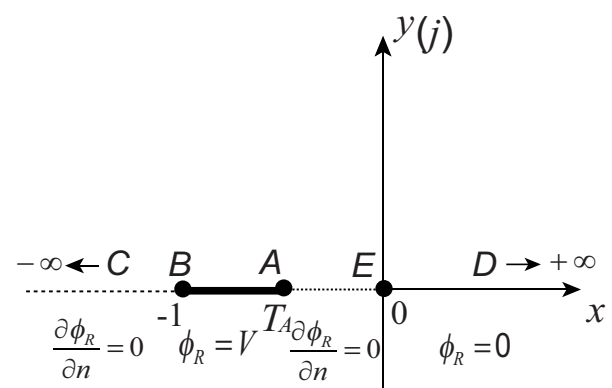

T-plane

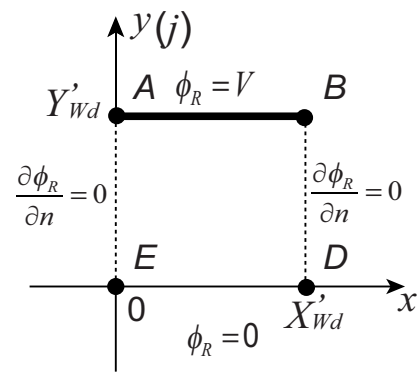

W-plane

FIG. 2. (a) Schematic of a 2D section of the DEP electrode showing the potentials. The vertical lines mark the period over which the system repeats. The rectangle $A B C D E$ is the basic cell for analysis. Also shown is the potential $\phi$, the potential phasor $\widetilde{\phi}$, and the value of the real part of the potential phasor $\phi_{R}$ on each electrode. The imaginary part of the potential phasor $\phi_{I}$ is zero everywhere. (b) Diagram showing the three complex planes used for Schwarz-Christoffel mapping (SCM) taking into account the lid. (c) Diagram showing the three complex planes used for the Schwarz-Christoffel mapping (SCM) procedure for array without the lid.

rameters of the system) are $Z_{A}=j g / 2, Z_{B}=j(w+g) / 2, Z_{C}=h$ $+j(w+g) / 2, Z_{D}=h$, and $Z_{E}=0$.

\section{B. SCM procedure for array with lid}

The interior of the polygon $A B C D E$ in the $Z$-plane is mapped into the upper half of the $T$-plane using the SCM method. The polygon $A B C D E$ is opened at point $F$ and the boundaries of the polygon mapped to the real axis of the $T$-plane. The coordinates of the corresponding points $Z_{A}$ to $Z_{E}$ in the $T$-plane are $T_{A}$ to $T_{E}$, respectively. The point $F$ is mapped to positive and negative infinity. The four interior angles of polygon $A B C D E$ at points $E, B, C, D$ are all $\pi / 2$. According to Eq. (3), the SCM integral from $T$-plane to $Z$-plane is given by

$$
\begin{aligned}
Z= & C_{1} \int^{T}\left(T-T_{E}\right)^{-1 / 2}\left(T-T_{B}\right)^{-1 / 2}\left(T-T_{C}\right)^{-1 / 2}\left(T-T_{D}\right)^{-1 / 2} d T \\
& +C_{2}
\end{aligned}
$$

where $Z=Z_{x}+j Z_{y}$ refers to the complex coordinate of any point in the interior of polygon $A B C D E$ in the $Z$-plane. $T$ $=T_{x}+j T_{y}$ refers to the complex coordinate of any point in the upper half of the $T$-plane. 
Since the SCM method allows up to three points to be arbitrarily chosen along the real axis of the $T$-plane, we fix the coordinates of $T_{D}=0, T_{C}=1$ and $T_{B}=1.2$ as shown in Fig. 2(b). For $T>T_{E}>T_{B}>T_{C}>T_{D}$, the solution of Eq. (4) is an elliptic integral [48]:

$$
\begin{aligned}
Z & =C_{3} F\left(\varpi_{d 1}, k_{d 1}\right)+C_{2} \\
& =C_{3} \int_{0}^{\lambda_{d 1}} \frac{d \lambda_{d 1}}{\left(1-\lambda_{d 1}^{2}\right)\left(1-k_{d 1}^{2} \lambda_{d 1}^{2}\right)} d \lambda_{d 1}+C_{2},
\end{aligned}
$$

where $F\left(\varpi_{d 1}, k_{d 1}\right)$ is the elliptical integral of the first kind and $k_{d 1}$ is the modulus of the elliptic function. The expressions for the variables $C_{3}, \varpi_{d 1}, k_{d 1}$, and $\lambda_{d 1}$ are given in Appendix B.

Equation (5) links the $T$-plane to the $Z$-plane. The values of the coefficients $C_{2}$ and $C_{3}$ can be solved by a mapping relationship between the coordinates of the corresponding points in the two planes, which is shown in Appendix B:

$$
C_{2}=0, \quad C_{3}=\frac{h}{K\left(k_{d 1}\right)}=\frac{w+g}{2 K\left(k_{d 1}^{\prime}\right)},
$$

where $K\left(k_{d 1}\right)$ is the complete elliptic integral of the first kind and $k_{d 1}^{\prime}=\sqrt{1-k_{d 1}^{2}}$. The expression for $C_{3}$ also provides the relationship between the complete elliptical integral and the geometrical parameters of the system:

$$
\frac{K\left(k_{d 1}\right)}{K\left(k^{\prime}{ }_{d 1}\right)}=\frac{2 h}{w+g} .
$$

The value of the modulus, $k_{d 1}$ can be calculated by inputting arbitrary geometrical parameters using Hilberg's approximation [49].

The inverse function of Eq. (5) enables us to express $T$ in terms of $Z$ :

$$
T=\frac{T_{E} T_{B} \mathrm{cn}^{2}\left(\frac{Z}{C_{3}}, k_{d 1}\right)}{T_{B}-T_{E} \operatorname{sn}^{2}\left(\frac{Z}{C_{3}}, k_{d 1}\right)},
$$

where $\operatorname{sn}(\ldots, \ldots)$ and $\operatorname{cn}(\ldots, \ldots)$ are the Jacobian elliptic functions. The expression for $T_{E}$ is given in Appendix B and $T_{B}=1.2$.

The second SCM is used to transform the upper half of the $T$-plane into a rectangle in the model plane ( $W$-plane). The electric field is uniformly distributed in the interior of the rectangle, due to the restriction from the transformed boundaries in the $W$-plane. The corresponding points are $W_{A}=j Y_{W d}, W_{B}=X_{W d}+j Y_{W d}, W_{D}=X_{W d}$, and $W_{E}=0$, where $X_{W d}$ and $Y_{W d}$ are the size of the rectangle along the real and imaginary axis, respectively. Similarly, the transformation from the $T$-plane to the $W$-plane is given by

$$
\begin{aligned}
W= & D_{1} \int^{T}\left(T-T_{E}\right)^{-1 / 2}\left(T-T_{A}\right)^{-1 / 2}\left(T-T_{B}\right)^{-1 / 2}\left(T-T_{D}\right)^{-1 / 2} d T \\
& +D_{2} .
\end{aligned}
$$

It should be noted that, compared to Eq. (4), in this transfor- mation point $A$ replaces $C$ to become an angle of the rectangle. The integral solution of Eq. (9) is

$$
\begin{aligned}
W & =D_{3} F\left(\varpi_{d 2}, k_{d 2}\right)+D_{2} \\
& =D_{3} \int_{0}^{\lambda_{d 2}} \frac{d \lambda_{d 2}}{\left(1-\lambda_{d 2}^{2}\right)\left(1-k_{d 2}^{2} \lambda_{d 2}^{2}\right)} d \lambda_{d 2}+D_{2} .
\end{aligned}
$$

The expressions for $D_{3}, \varpi_{d 2}, k_{d 2}$, and $\lambda_{d 2}$ are given in Appendix B. Equation (10) links the $T$-plane with the $W$-plane. The values of the coefficients $D_{2}$ and $D_{3}$ can be obtained by a similar procedure to the first SCM performance:

$$
D_{2}=0, \quad D_{3}=\frac{X_{W d}}{K\left(k_{d 2}\right)}=\frac{Y_{W d}}{K\left(k_{d 2}^{\prime}\right)} .
$$

\section{Analytical electric field solution in array with lid}

Since Laplace's equation remains invariant under conformal mapping, the potential gradients in the physical plane, $\nabla \phi_{Z}$, and model plane, $\nabla \phi_{W}$, are related by [31]

$$
\nabla \phi_{Z}=\nabla \phi_{W} \overline{f^{\prime}(Z)}=\nabla \phi_{W} \frac{\overline{d W}}{d Z}
$$

$\overline{f^{\prime}(Z)}$ is the conjugate of the derivative of $f(Z)$, which is the linking transformation equation between the $Z$ - and $W$-planes. Using this relationship and combining Eqs. (4) and (9), the nonuniform electric field distribution in the $Z$-plane, $\mathbf{E}_{Z d}$, can be derived as

$$
\begin{aligned}
\mathbf{E}_{Z d}=-\nabla \phi_{Z d} & =-\nabla \phi_{W d} \overline{\left(\frac{d W}{d T} \frac{d T}{d Z}\right)} \\
& =j \frac{V \frac{K\left(k_{d 1}\right)}{h}\left[\frac{T_{A}\left(T_{E}-T_{B}\right)\left(T-T_{C}\right)}{T_{B}\left(T_{E}-T_{C}\right)\left(T-T_{A}\right)}\right]^{1 / 2}}{,}
\end{aligned}
$$

where $\phi_{Z d}$ and $\phi_{W d}$ are the potentials in the $Z$ - and $W$-planes, respectively, and $V$ is the potential difference between the electrode $A B$ and the axis of odd symmetry, $D E$. Note that the expressions for $T_{A}$ and $T_{E}$ are given in Appendix B and $T_{B}=1.2$ and $T_{C}=1$.

Equation (13) is the analytical solution for the electric field in the basic cell for the interdigitated DEP array. Compared to the previous analytical solution using series expansions, Eq. (13) is significantly simpler. The features of the electric field distribution that are governed by the geometry of the device are clearly identified. The electric field magnitude approaches zero at point $C$ (when $T=T_{C}$ ) and infinity at point $A$ (when $T=T_{A}$ ), the edge of the electrode.

Substituting Eq. (8) into (13), we obtain the electric field expression as a function of position in the interior of polygon $A B C D E$ in the $Z$-plane: 


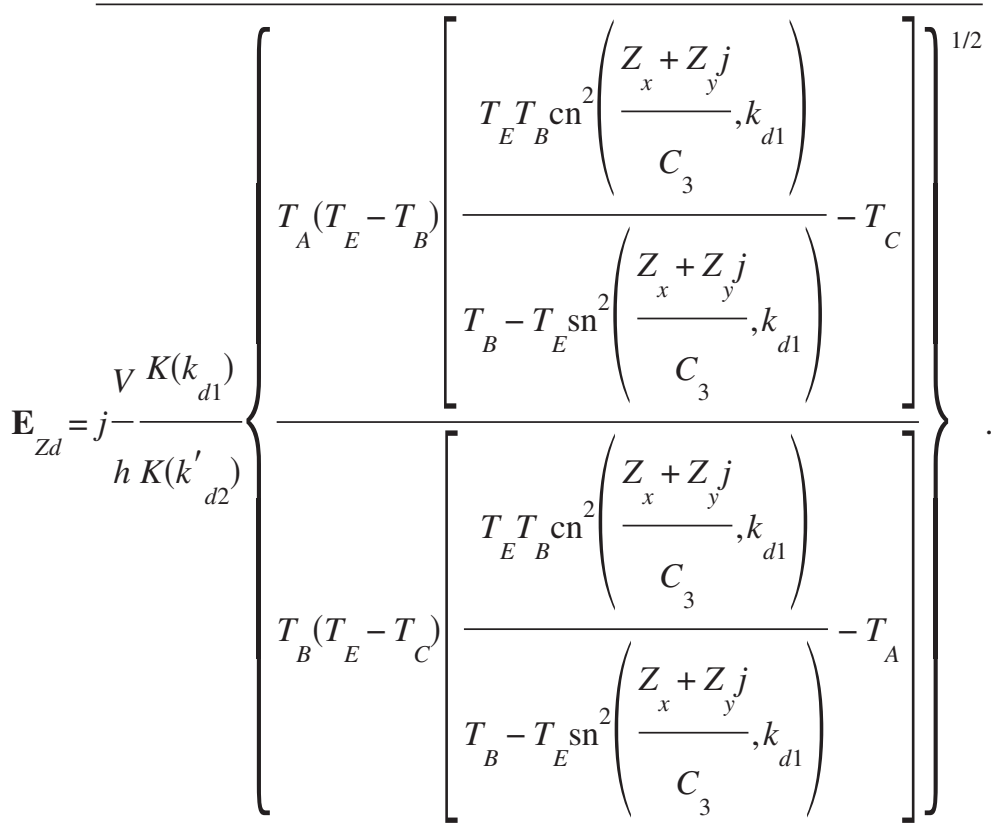

To calculate the DEP force in the array, we determine the value of the dielectrophoretic force component, $\boldsymbol{\nabla}|\widetilde{\mathbf{E}}|^{2}$ :

$$
\boldsymbol{\nabla}\left|\mathbf{E}_{Z d}\right|^{2}=\boldsymbol{\nabla}\left(\mathbf{E}_{Z d_{-} x}^{2}+\mathbf{E}_{Z d_{-} y}^{2}\right),
$$

where $\mathbf{E}_{Z d_{-} x}$ and $\mathbf{E}_{Z d_{-} y}$ represent the $x$ and $y$ components of the electric field in the real geometry, respectively.

Separating the real and imaginary parts of Eq. (15) to give these components requires the formulas for the Jacobian elliptic functions [50] (see Appendix B).

\section{Analytical electric field solution in array without lid}

One common feature of previous analytical solutions [23-27] is that the upper boundary, i.e., the lid of the channel, was not considered in the analysis. Instead, the potential was assumed to tend to zero as the height goes to infinity, which is only valid if the lid is far from the electrodes. However if the height of the channel is comparable to the width of the electrodes (and gap), the lid will influence the electric field distribution.

Equation (14) gives the field distribution for a fixed channel height. For comparison with existing solutions we also use the SCM method to derive the electric field solution for a system without the lid.

The three complex planes for this case are shown in Fig. 2(c). The boundary conditions are the same as for the previous case, except for the top Neumann boundary condition. In the $Z$-plane, the coordinates of each point are $Z_{A}=w / 2, Z_{B}$ $=0, Z_{C}=0+j \infty, Z_{D}=(w+g) / 2+j \infty$, and $Z_{E}=(g+w) / 2$. The SCM integral from the $Z$-plane to the $T$-plane is

$$
Z=C_{1}^{\prime} \int^{T}\left(T-T_{E}\right)^{-1 / 2}\left(T-T_{B}\right)^{-1 / 2} d T+C_{2}^{\prime} .
$$

In the $T$-plane, the coordinates of point $B$ and $E$ are chosen at: $T_{B}=-1$ and $T_{E}=0$. The points $C$ and $D$ are mapped to negative and positive infinity on the real axis of the $T$-plane respectively. The solution of Eq. (16) is

$$
Z=2 C_{1}^{\prime} \ln (\sqrt{T}+\sqrt{T+1})+C_{2}^{\prime},
$$

where the coefficients $C_{1}^{\prime}$ and $C_{2}^{\prime}$ are obtained from applying the mapping to points $B$ and $E$ in the $Z$ - and $T$-planes:

$$
C_{1}^{\prime}=j \frac{w+g}{2 \pi}, \quad C_{2}^{\prime}=\frac{w+g}{2} .
$$

The inverse function of Eq. (17) is

$$
T=\sinh ^{2}\left(\frac{Z-C_{2}^{\prime}}{2 C_{1}^{\prime}}\right) .
$$

This gives the coordinate of point $A$ in the $T$-plane:

$$
T_{A}=\sinh ^{2}\left(j \frac{g \pi}{2(w+g)}\right) .
$$

The SCM integral from the $T$-plane to the $W$-plane is

$$
W=D_{1}^{\prime} \int^{T}\left(T-T_{E}\right)^{-1 / 2}\left(T-T_{A}\right)^{-1 / 2}\left(T-T_{B}\right)^{-1 / 2} d T+D_{2}^{\prime} .
$$

For $T>T_{E}>T_{A}>T_{B}$ the solution of Eq. (21) is an elliptic integral [48]: 


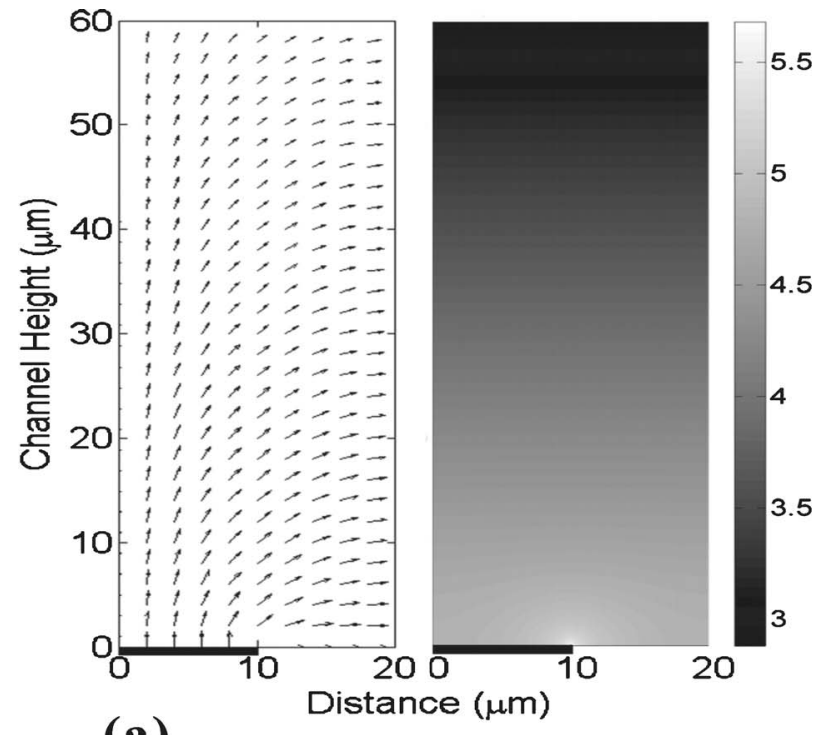

(a)

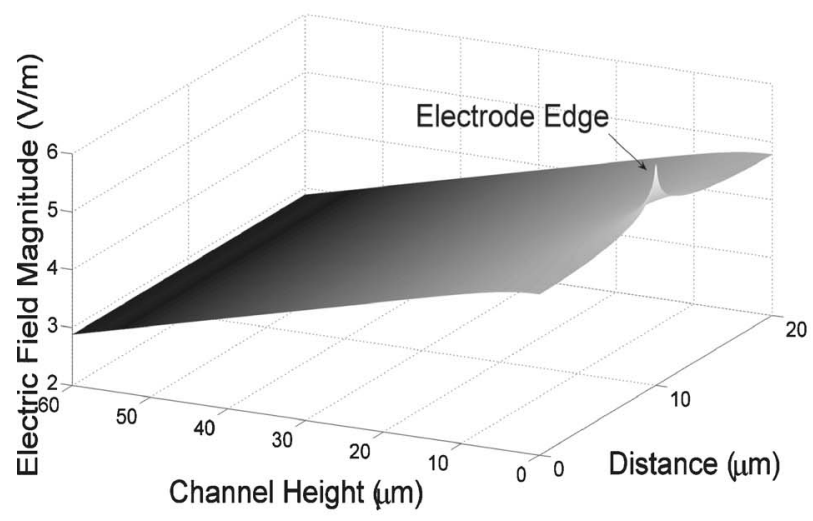

(b)
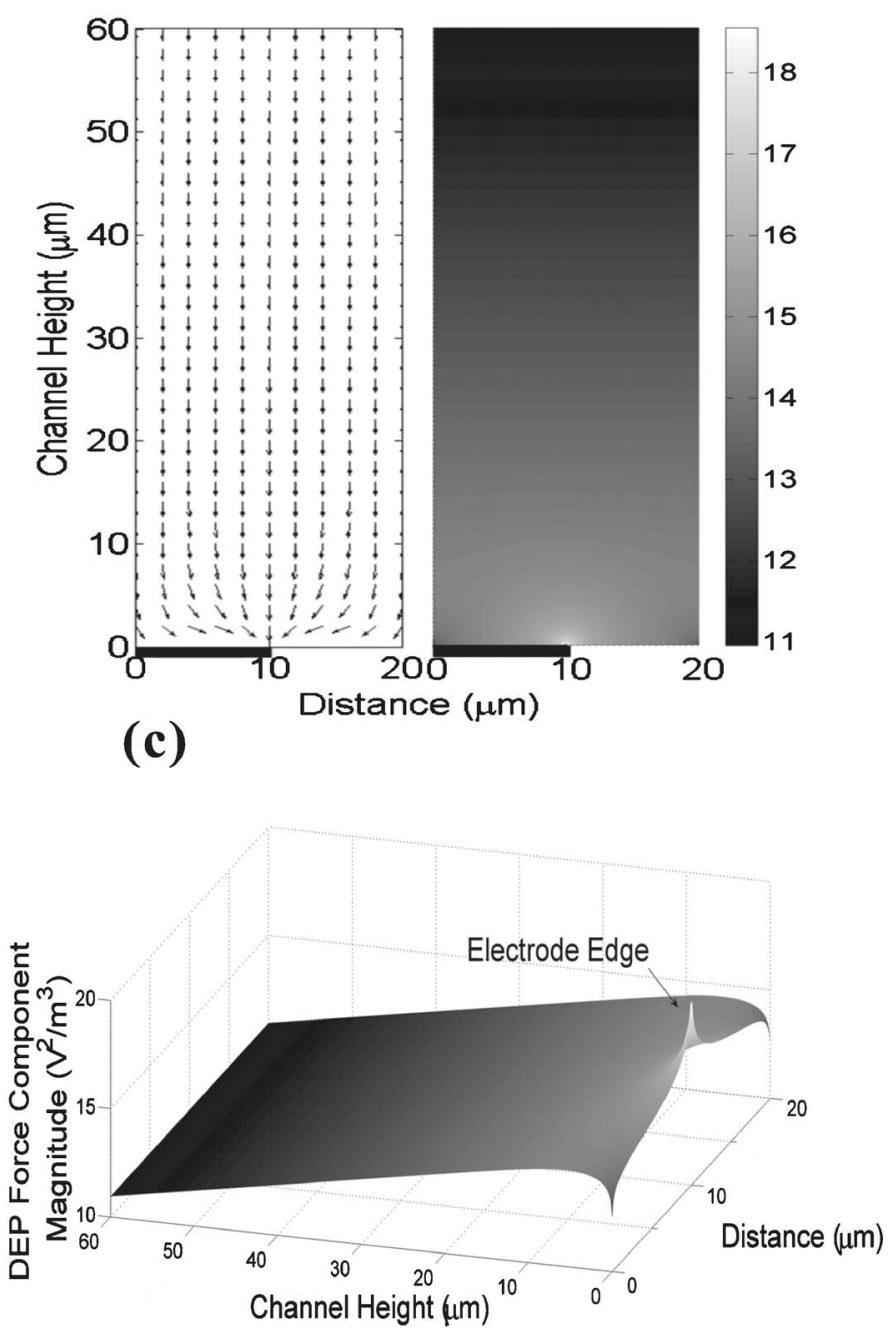

(d)

FIG. 3. The electric field and DEP force component $\left(\boldsymbol{\nabla}\left|\mathbf{E}_{Z d}\right|^{2}\right)$ in the basic cell of the DEP array without lid. The positions of the electrodes are drawn on the figures. (a) Electric field vector and magnitude (V/m, $\log _{10}$ scale), (b) 3D surface plot of electric field magnitude, (c) DEP force component vector and magnitude ( $\log _{10}$ scale), and (d) 3D surface plot of $\nabla\left|\mathbf{E}_{Z d}\right|^{2}$.

$$
\begin{aligned}
W & =D_{3}^{\prime} F\left(\varpi_{d 3}, k_{d 3}\right)+D_{2}^{\prime} \\
& =D_{3}^{\prime} \int_{0}^{\lambda_{d 3}} \frac{d \lambda_{d 3}}{\left(1-\lambda_{d 3}^{2}\right)\left(1-k_{d 3}^{2} \lambda_{d 3}^{2}\right)} d \lambda_{d 3}+D_{2}^{\prime}
\end{aligned}
$$

with

$$
\begin{gathered}
D_{3}^{\prime}=\frac{2 D_{1}^{\prime}}{\sqrt{T_{E}-T_{B}}}, \quad \varpi_{d 3}=\arcsin \left(\sqrt{\frac{T-T_{E}}{T-T_{A}}}\right), \\
k_{d 3}=\sqrt{\frac{T_{A}-T_{B}}{T_{E}-T_{B}}}, \quad \lambda_{d 3}=\sin \varpi_{d 3} .
\end{gathered}
$$

In the $W$-plane, $W_{A}=j Y^{\prime}{ }_{W d}, W_{B}=X^{\prime}{ }_{W d}+j Y^{\prime}{ }_{W d}, W_{D}=X^{\prime}{ }_{W d}$, and $W_{E}=0$. The values of the coefficients $D^{\prime}{ }_{2}$ and $D^{\prime}{ }_{3}$ are

$$
D_{2}^{\prime}=0, \quad D_{3}^{\prime}=\frac{X_{W d}^{\prime}}{K\left(k_{d 3}\right)}=\frac{Y_{W d}^{\prime}}{K\left(k_{d 3}^{\prime}\right)} .
$$

The nonuniform electric field distribution in the $Z$-plane is then solved as

$$
\mathbf{E}_{Z d}^{\prime}=-\nabla \phi_{W d} \overline{\left(\frac{d W}{d T} \frac{d T}{d Z}\right)}=\overline{\frac{\pi V}{w+g} \frac{1}{K\left(k^{\prime}{ }_{d 3}\right)}\left(\frac{T_{E}-T_{B}}{T-T_{A}}\right)^{1 / 2}} .
$$

This is the analytical solution for the electric field in the basic cell of the DEP electrode array without the insulating lid. Note that $T_{A}$ is given by Eq. (20) and $T_{B}=-1$ and $T_{E}$ $=0$. Compared to Eq. (13), the electric field magnitude goes to infinity at the edge of the electrodes (point $A$ ). However there is no point of zero field, since point $C$ goes to infinity in the $y$ direction in the $Z$-plane. In this paper, calculations of the electric fields and $\boldsymbol{\nabla}\left|\mathbf{E}_{Z d}\right|^{2}$ using Elliptic functions were 


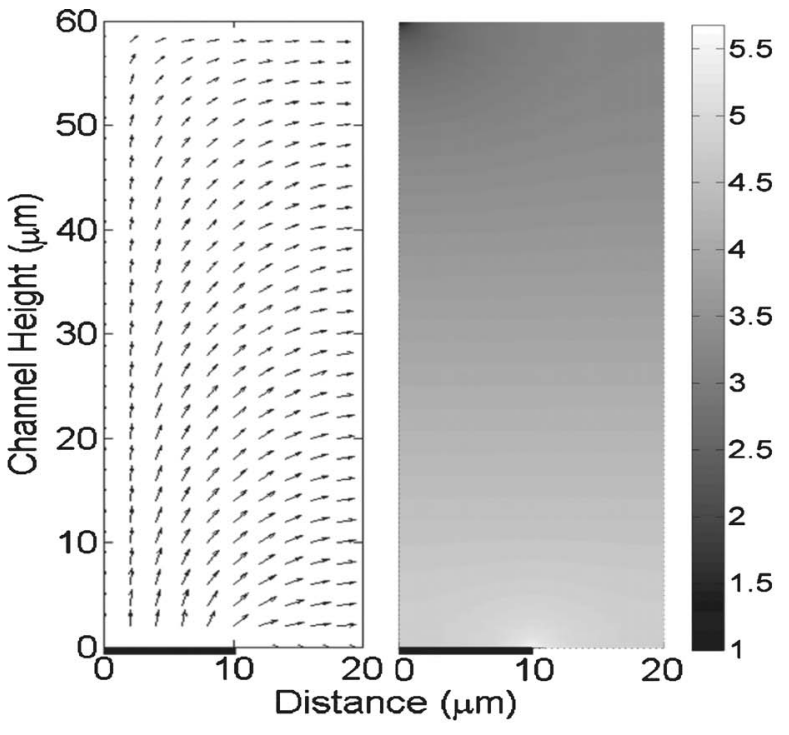

(a)

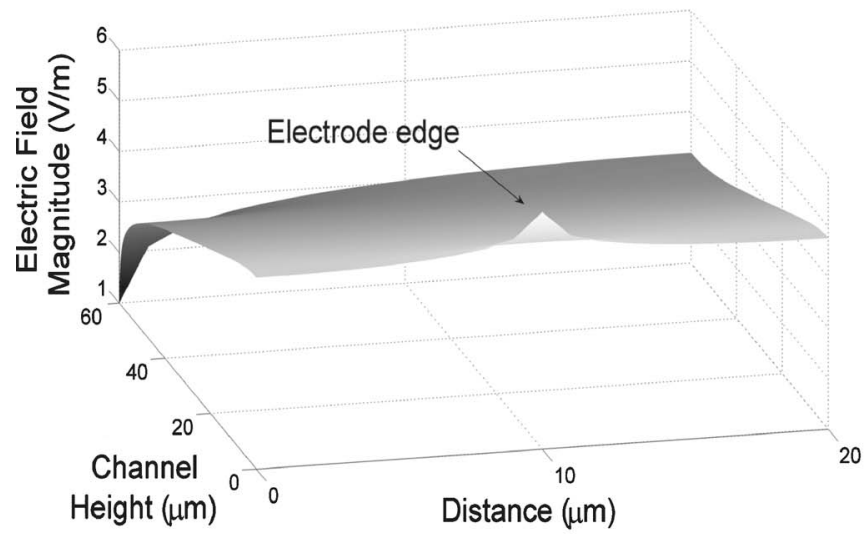

(b)

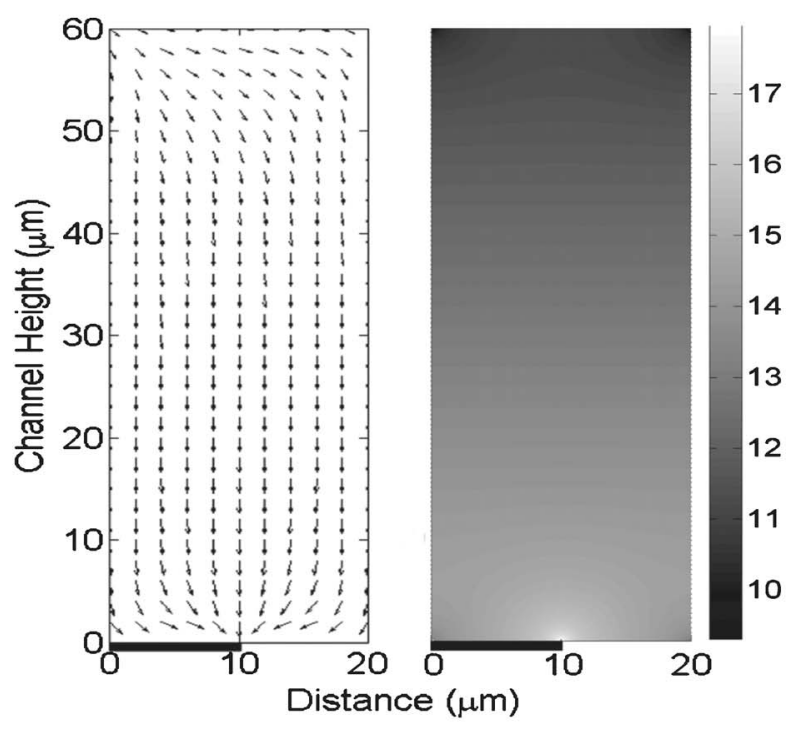

(c)

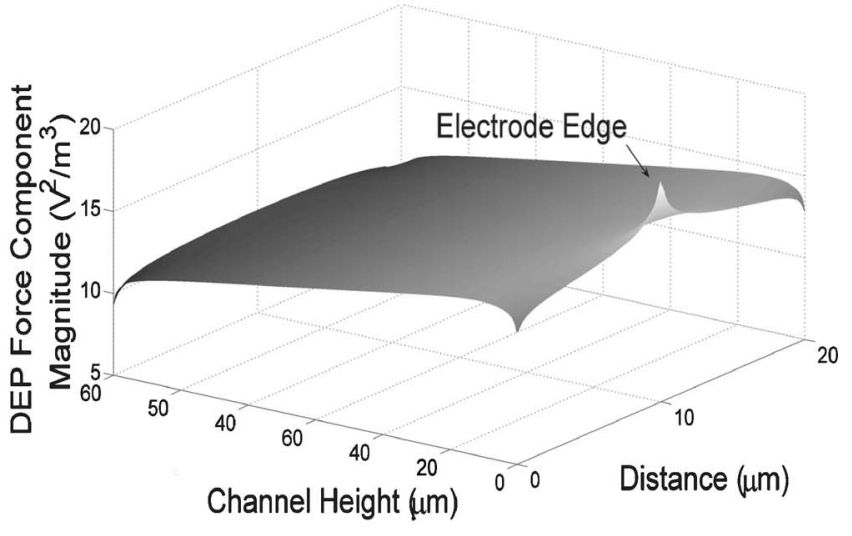

(d)

FIG. 4. Solutions of the electric field and DEP force component $\left(\nabla\left|\mathbf{E}_{Z d}\right|^{2}\right)$ in the basic cell of the DEP array with the lid. (a) Electric field vector and magnitude $\left(\mathrm{V} / \mathrm{m}, \log _{10}\right.$ scale), (b) 3D surface plot of the magnitude of the electric field, (c) DEP force component, vector, and magnitude ( $\log _{10}$ scale), and (d) 3D surface plot of $\boldsymbol{\nabla}\left|\mathbf{E}_{Z d}\right|^{2}$.

performed in MATLAB ${ }^{\mathrm{TM}}$ (Mathworks Inc, Natick, MA, USA).

\section{E. Electric field distribution and DEP force}

Typical experimental values were used in the calculations: The electrode width and gap were $20 \mu \mathrm{m}$ and the voltage applied to the electrode was $1 \mathrm{~V}$. For the case of the array with lid, the channel height was $60 \mu \mathrm{m}$.

Figure 3(a) shows the electric field up to $60 \mu \mathrm{m}$ in height, for the basic cell without the lid. The magnitude of the electric field is symmetrical about the vertical line through the electrode edge, since the electrode width and the gap are the same. Figure 3(b) shows the electric field magnitude as a 3D surface, clearly demonstrating the maximum (theoretically infinity) at the electrode edge and the exponential decrease with height in the far field, as reported previously $[26,27,30]$.
Figure 3(c) is a plot of $\boldsymbol{\nabla}\left|\mathbf{E}_{Z d}\right|^{2}$, showing that the DEP force (direction and magnitude) is symmetrical about the vertical line through the electrode edge, also due to the same length of the electrode width and the gap. It can also be seen that in the far field, the DEP force acts only in the vertical direction and in the near field, $\boldsymbol{\nabla}\left|\mathbf{E}_{Z d}\right|^{2}$ points towards the electrode edge. Figure 3(d) shows the magnitude of $\nabla\left|\mathbf{E}_{Z d}\right|^{2}$ as a 3D surface, clearly showing the maximum at the electrode edge, the minima in the corners at the substrate and the exponential decrease with height in the far field.

Figure 4(a) shows the electric field for the array with the lid. The bending of the electric field lines by the lid is clearly observed in the vector plot, where the vectors near the top boundary tend to lie parallel to the lid. This clearly shows the consequence of the Neumann boundary condition, imposed at the lid. In this case, the magnitude of the electric field is not symmetrical about the vertical line through the electrode 

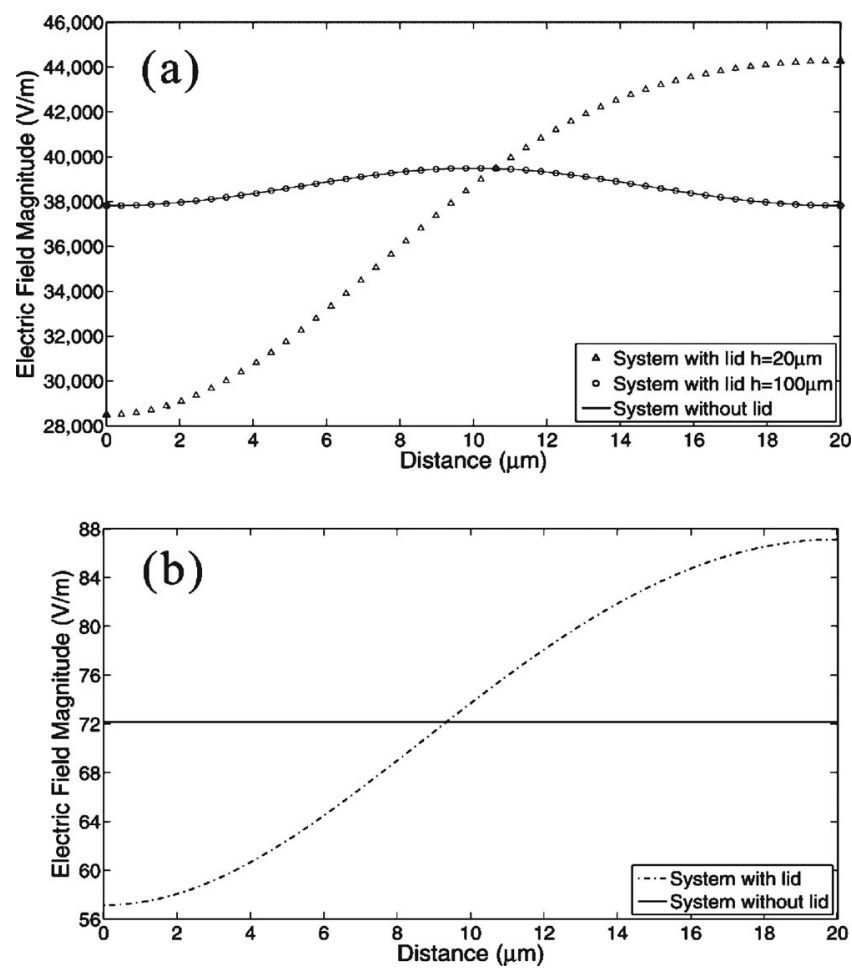

FIG. 5. Plot showing the influence of the lid on the electric field distribution for an electrode array with width and gap set to $20 \mu \mathrm{m}$. (a) Near field region for two cases: channel height $20 \mu \mathrm{m}$ or $100 \mu \mathrm{m}$. (b) Far field region at $90 \mu \mathrm{m}$ for a channel height of $100 \mu \mathrm{m}$.

edge, even though the electrode width and the gap are equal. This can be seen more clearly in Fig. 4(b), the electric field magnitude is zero at point $C$ (the point where the lid meets the even symmetry axis), since at this point, it has to simultaneously satisfy the Neumann boundary condition at the lid and the even symmetry axis $[C D$ and $B C$ in Fig. 2(b), $Z$-plane]. Close to the electrode surface, there is a maximum at the electrode edge. Figure 4(c) shows $\boldsymbol{\nabla}\left|\mathbf{E}_{Z d}\right|^{2}$ for this case, demonstrating that while the DEP force in the near field is approximately the same as for the previous case, in the far field (close to the lid), the behavior is quite different. The field minimum in the corner has a strong influence on the direction of the DEP force, which is no longer only in the vertical direction, and practically would result in a negative DEP trap at point $C$. Figure 4(d) shows the maximum in the DEP force at the electrode edge and the four minima in each corner.

Since most analytical solutions ignore the presence of an upper boundary, a further examination of the effect of the lid is warranted. If the channel height is of the same order of magnitude as the electrode width and gap distance, the effect of the lid is always important. Figure 5(a) shows significant difference in the electric field magnitude at a height of $10 \mu \mathrm{m}$ for two different channel heights, with the solution for a channel height of $20 \mu \mathrm{m}$ showing considerable disagreement with the no lid case. The $100 \mu \mathrm{m}$ channel height case agrees with the no lid situation, showing that the effect of the lid in the near field region becomes negligible as the channel height increases. However, this effect still plays an important role in the far field region, near the top of the channel. This is demonstrated by Fig. 5(b), which shows the electric field magnitude at a height of $90 \mu \mathrm{m}$ for the $100 \mu \mathrm{m}$ high channel compared with the solution for no lid, where the magnitude of the field is constant. This figure shows that the presence of the lid cannot be ignored when determining the DEP force in the region close to the lid.

\section{F. Comparisons with FEM simulations-electric field}

The analytical solution for the electric field was compared with numerical simulations performed using COMSOL MULTIPHYSICS $^{\mathrm{TM}}$ (Comsol Ltd.), a finite element method (FEM) solver. In order to ensure the accuracy of the numerical simulations, the meshing was increased around the electrode edge (point $A$ ), and along paths where the electric field was sampled. The analytical solutions were validated more comprehensively by varying the ratio of the electrode width and gap distance. For the array with lid, the height of the channel was set to $60 \mu \mathrm{m}$, with the sum of electrode width and gap fixed at $40 \mu \mathrm{m}$. The ratios of electrode:gap width were $1: 3,1: 1$, and $3: 1$ and comparisons of the electric field were performed at heights of $10 \mu \mathrm{m}$ (near field region), $30 \mu \mathrm{m}$ (middle field region), and $50 \mu \mathrm{m}$ (far field region), respectively.

Figures 6(a)-6(c) demonstrate excellent agreement between the SCM solutions and the FEM solutions for the three ratios and heights detailed above. The figures also demonstrate the relationship between the electric field magnitude and the ratio of the electrode width and gap. In the near field region, Fig. 6(a), the position of the electrode edge determines the region of maximum field. By extending the edge of the electrode towards the odd symmetry axis $(D E)$, the high field region shifts from the even symmetry axis $(B C)$ side to the odd symmetry axis side. In the middle and far field regions [Figs. 6(b) and 6(c)], this effect gradually disappears and the electric field magnitude on the odd symmetry axis side is always higher than that on the even symmetry axis side, due to the existence of the zero field point. For the array without lid, comparisons were performed for the same electrode width to gap ratios, with the channel height set to $500 \mu \mathrm{m}$ in the FEM simulations. The values of the electric field are sampled in the near field region at a height of $10 \mu \mathrm{m}$ and the results are shown in Fig. 6(d), again showing excellent agreements with the analytical model.

\section{G. Comparisons with Fourier series solutions}

The results of the comparison of the SCM solutions of $\boldsymbol{\nabla}\left|\mathbf{E}_{Z d}\right|^{2}$ (the DEP force component) with the simplified closed form Fourier series (FOU) solution [27] are shown in Figs. 7(a)-7(d). The electrode width and gap were both set to $20 \mu \mathrm{m}$. In Fig. 7(a), the magnitude of $\boldsymbol{\nabla}\left|\mathbf{E}_{Z d}\right|^{2}$ is plotted along the vertical line through the edge of the electrode, demonstrating that the FOU and SCM solutions only seem to agree at intermediate heights (between $10 \mu \mathrm{m}$ to $50 \mu \mathrm{m}$ ), where the magnitude of the force decreases exponentially. In the far field region, the FOU and SCM solutions without lid agree but both disagree with the SCM lid solution, as ex- 

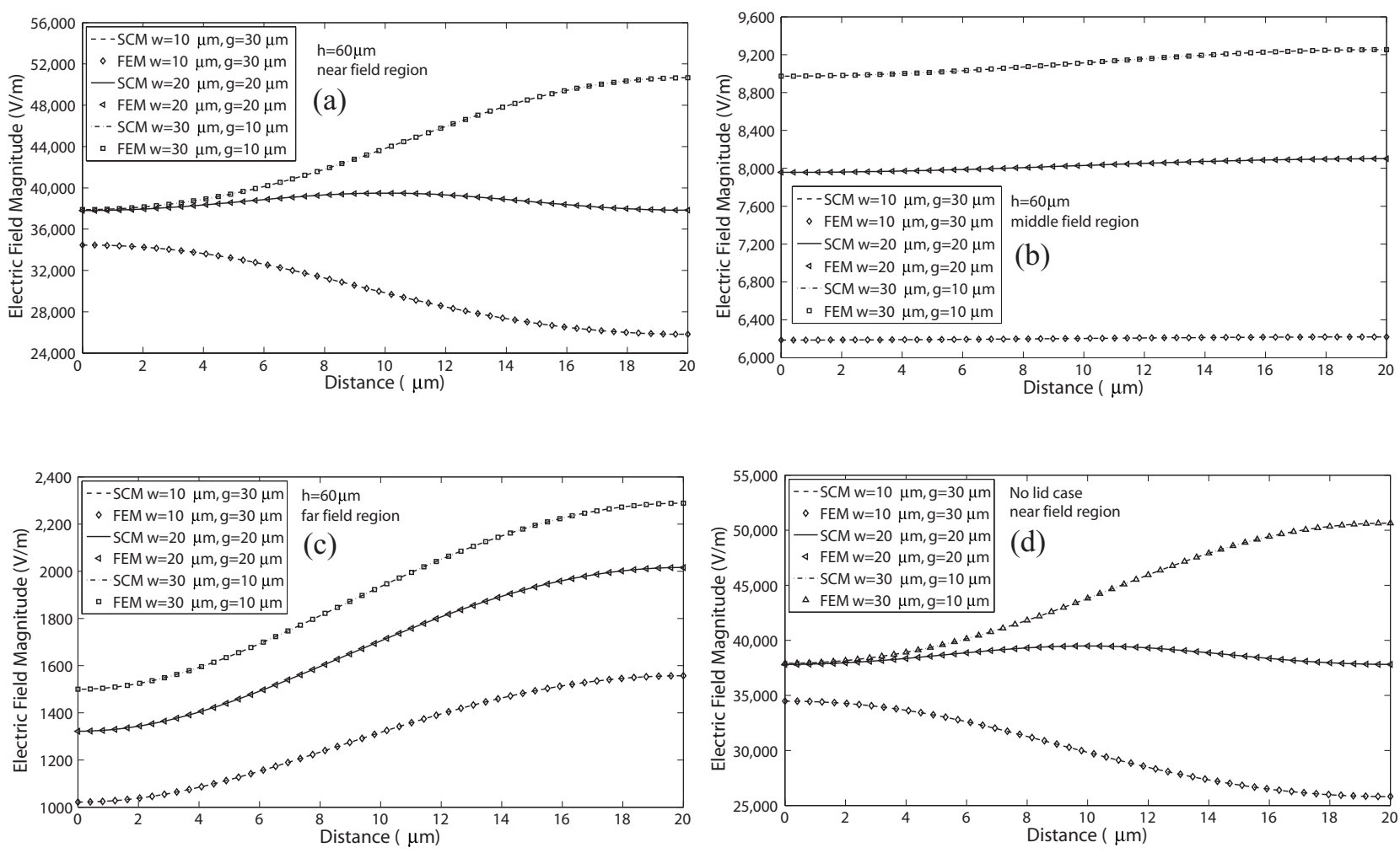

FIG. 6. Plot showing the electric field distribution from the SCM solutions and FEM simulations for different geometries. (a) Near field region, with lid (b) midfield region, with lid (c) far field region, with lid (d) near field region, no lid.
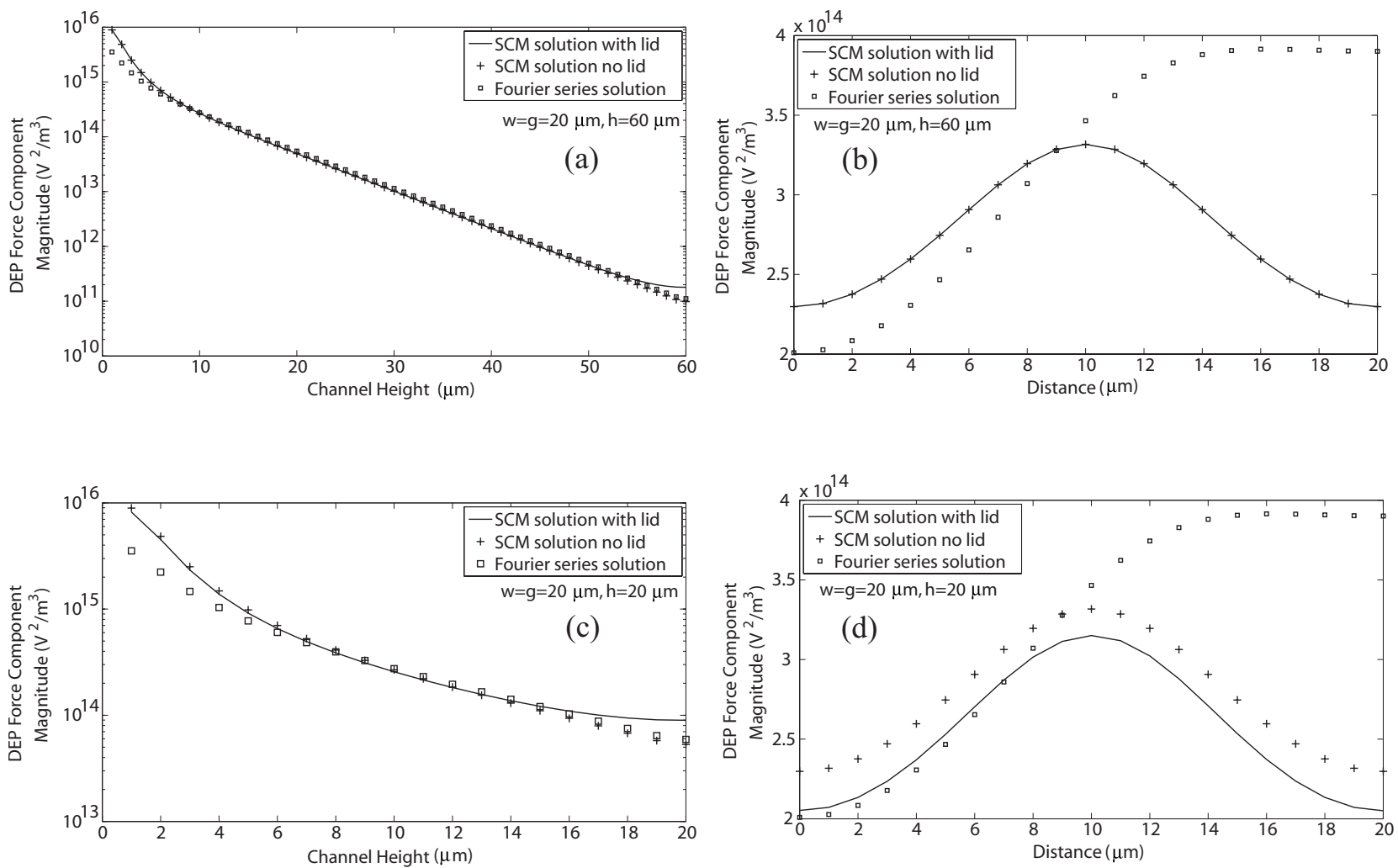

FIG. 7. Comparison of the DEP force component $\left(\nabla\left|\mathbf{E}_{Z d}\right|^{2}\right)$ calculated by the SCM and Fourier series solutions. (a) Magnitude calculated along a vertical line through the edge of the electrode, $60 \mu \mathrm{m}$ channel height; (b) Magnitude calculated along a horizontal line at a height of $10 \mu \mathrm{m}, 60 \mu \mathrm{m}$ channel height; (c) Magnitude calculated along a vertical line through the edge of the electrode, $20 \mu \mathrm{m}$ channel height; (d) Magnitude calculated along the horizontal line at height of $10 \mu \mathrm{m}, 20 \mu \mathrm{m}$ channel height. 

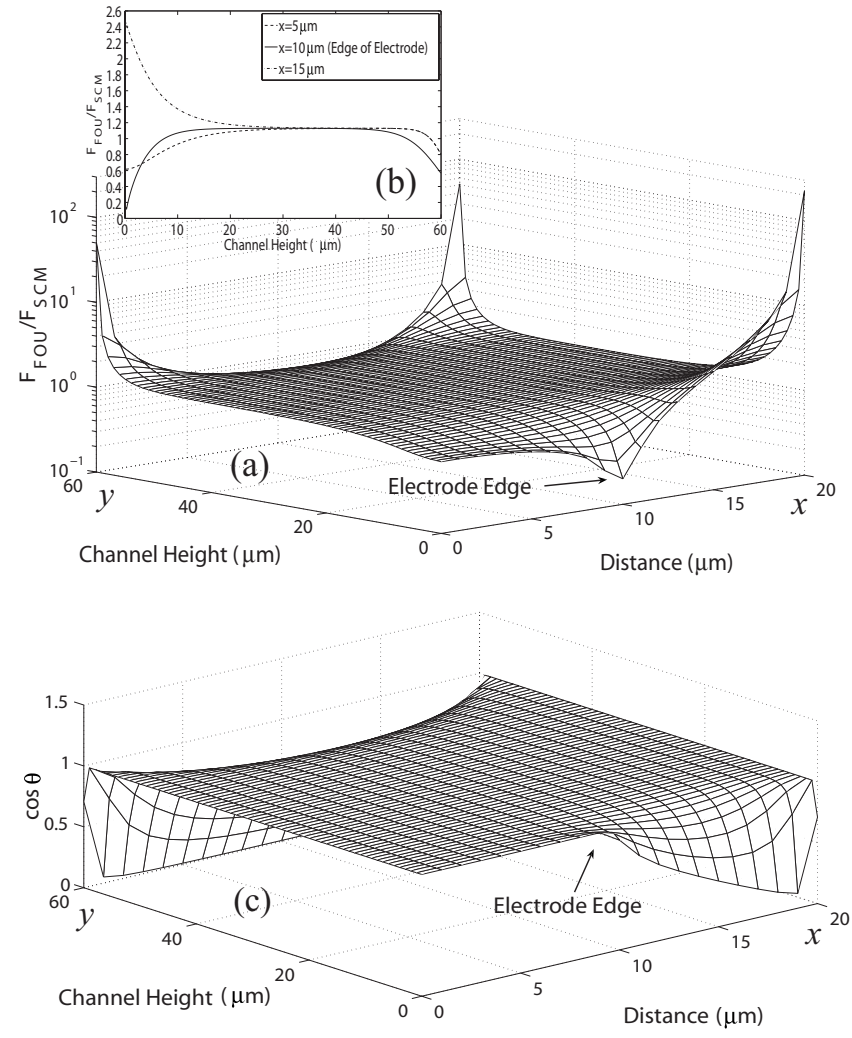

FIG. 8. (a) Surface plot of the magnitude of the ratio of the DEP force from the Fourier solution $\left(F_{\mathrm{FOU}}\right)$ and SCM solution $\left(F_{\mathrm{SCM}}\right)$, with lid, for $60 \mu \mathrm{m}$ high channel, $20 \mu \mathrm{m}$ electrode width and gap. (b) Plot of the value of the magnitude of the ratio $\left(F_{\mathrm{FOU}} / F_{\mathrm{SCM}}\right)$ along three vertical lines $(x=5,10$, and $15 \mu \mathrm{m})$. (c) Surface plot of the deviation of the direction $(\cos \theta)$ between the DEP force from the Fourier solution $\left(F_{\mathrm{FOU}}\right)$ and SCM solution $\left(F_{\mathrm{SCM}}\right)$, with lid. The alignment of the two solutions is only achieved, when $\theta=0^{\circ}$, leading to $\cos \theta=1$.

pected due to the neglected upper boundary condition. In the near field solution, the SCM solutions with and without lid agree with each other but deviation of the FOU solution is readily apparent. Calculation of the magnitude of $\nabla\left|\mathbf{E}_{Z d}\right|^{2}$ along a horizontal line at a height of $10 \mu \mathrm{m}$, Fig. 7(b), shows that the FOU solution is not symmetrical about the vertical line through the edge of the electrode as observed in the vector plot in Fig. 5 of [27]. For low channels, the FOU solution is even more inaccurate, since the effect of the lid is more pronounced, as shown in Figs. 7(c) and 7(d).

A quantitative discussion of the difference between the two solution methods can be performed by examining the difference in magnitude and the angle of deviation in direction. The magnitude difference was determined as the ratio: $F_{\text {FOU }} / F_{\text {SCM }}$, where $F_{\text {FOU }}$ and $F_{\text {SCM }}$ are the magnitude of the DEP forces for the two solutions. The deviation between the directions of the force vectors was characterized by $\cos \theta$, defined as

$$
\cos \theta=\frac{F_{\mathrm{FOU}} \cdot F_{\mathrm{SCM}}}{\left|F_{\mathrm{FOU}}\right|\left|F_{\mathrm{SCM}}\right|} .
$$

Figure $8(a)$ shows a surface plot of the magnitude of
$F_{\mathrm{FOU}} / F_{\mathrm{SCM}}$. At the electrode edge, the magnitude of the DEP force from the Fourier series is approximately one order of magnitude less than that given by the SCM solution. However, at the three corners of the basic cell (except the corner on the surface of the electrode), the Fourier series solution is approximately two orders of magnitude greater than the SCM solution. In the mid range of height, Fig. 8(b) shows that even where the agreement was apparently good in Fig. 7 (a), the difference is still approximately $15 \%$. Figure 8(c) shows the surface plot of $\cos \theta$, demonstrating the misalignment between the direction of the DEP force in the FOU and SCM solutions in the far field region (close to the lid) and the near field region in the gap.

\section{TRAVELING WAVE DIELECTROPHORETIC ELECTRODE ARRAY}

In the twDEP electrode array, since the four signals are phase shifted by $90^{\circ}$ and are connected alternately to the electrodes, both the real and imaginary parts of the potential phasor must be considered.

\section{A. Boundary conditions}

Apart from the common boundary conditions, the values for the real and imaginary parts of the potential phasor at every electrode and the additional boundary conditions are shown in Fig. 9(a). The basic cell $(A B C D E F)$ chosen for analyzing the electric field distribution covers the region between the centers of two adjacent electrodes and the entire gap between. According to Fig. 9(a), the boundary conditions for the imaginary part of the potential phasor, $\phi_{I}$, are the mirror image of those for the real part, $\phi_{R}$, about the center of the gap. This indicates that only the real component of the potential, i.e., for $\mathbf{E}_{R}$, must be solved, which can then be transformed to give the solution for the imaginary component.

Figure 9(b) shows the three complex planes used in the SCM method to solve the real part of the electric field. In the $Z$-plane, which represents the real system, the basic cell $A B C D E F$ is rotated $90^{\circ}$. The complete boundary conditions for the real part of the potential phasor, $\phi_{R}$, are as shown. The Neumann boundary condition $\partial \phi_{R} / \partial n=0$ (indicated by dashed lines) holds along the insulating walls $A F$ and $C D$ as well as the axis of even symmetry, $B C$. Dirichlet boundary conditions define the fixed potential $\phi_{R}=V$ on the electrode $A B$ and $\phi_{R}=0$ on the electrode $E F$, as well as $\phi_{R}=0$ on the axis of odd symmetry, $D E$. The coordinates of each point in the physical plane are $Z_{A}=j(2 g+w) / 2, Z_{B}=j(w+g), Z_{C}=h$ $+j(w+g), Z_{D}=h, Z_{E}=0$, and $Z_{F}=j w / 2$.

\section{B. SCM procedure}

In the auxiliary plane (the $T$-plane), the polygon $A B C D E F$ is opened at point $G$ in the $Z$-plane and mapped into the upper half of the $T$-plane, with all the boundaries mapped onto the real axis. The coordinates of the corresponding points to $Z_{A}, \ldots, Z_{F}$ in the $T$-plane are $T_{A}, \ldots, T_{F}$, respectively. The point $G$ is mapped to positive and negative 

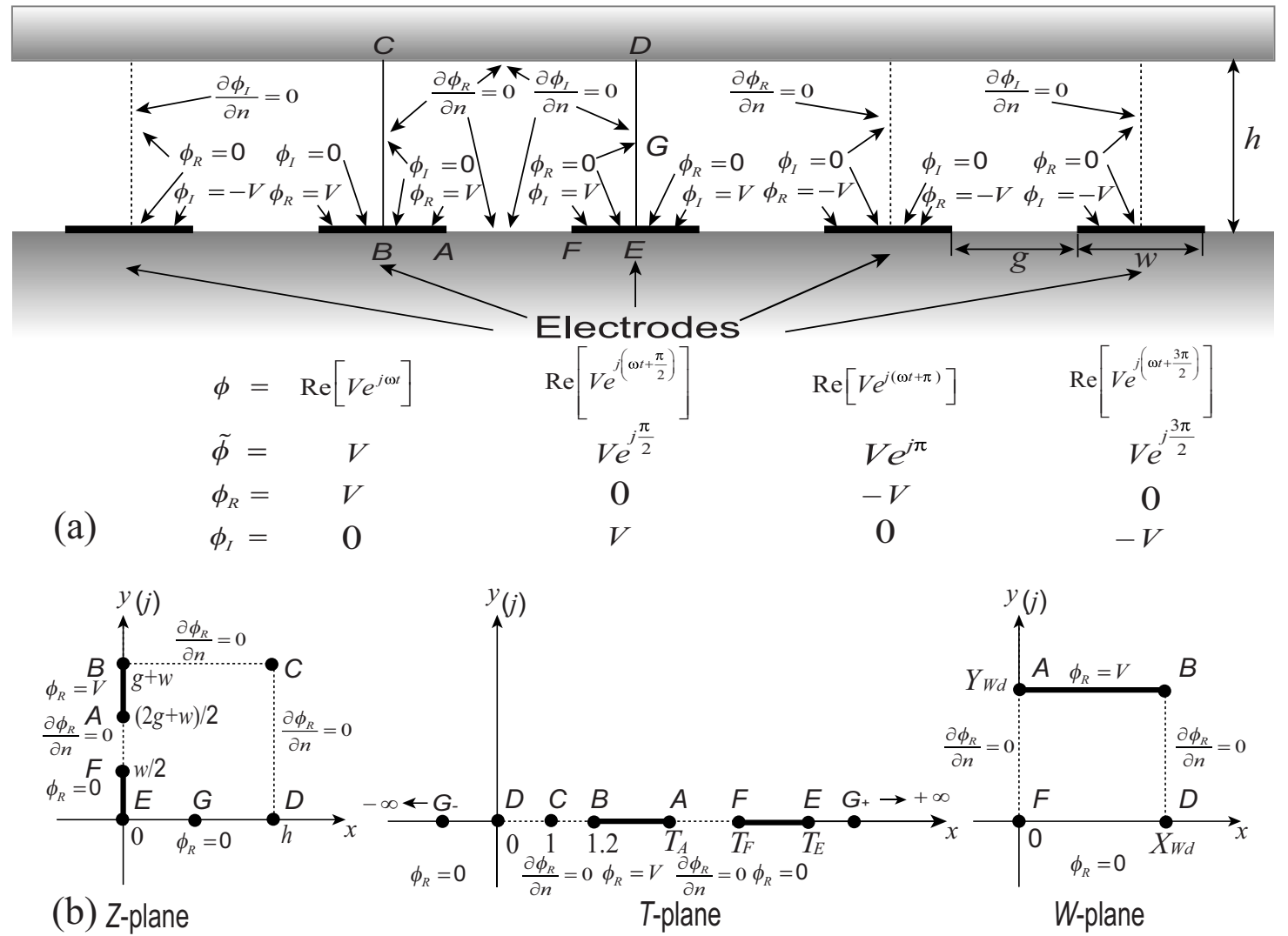

FIG. 9. (a) A schematic diagram showing a $2 \mathrm{D}$ section of the twDEP electrode array. The vertical lines mark the repeat distance. The rectangle $A B C D E F$ is the unit cell used in the analysis. Also shown are the values of the potential $\phi$, the potential phasor $\widetilde{\phi}$ and the value of the real and imaginary part of the potential phasor on each electrode. (b) Diagram showing the three complex planes used in the Schwarz-Christoffel mapping (SCM) procedure.

infinity. The four interior angles of polygon $A B C D E F$ at points $E, B, C$, and $D$ are all $\pi / 2$. The SCM integral from the $T$-plane to the $Z$-plane is given by

$$
\begin{aligned}
Z= & C_{1} \int^{T}\left(T-T_{E}\right)^{-1 / 2}\left(T-T_{B}\right)^{-1 / 2}\left(T-T_{C}\right)^{-1 / 2}\left(T-T_{D}\right)^{-1 / 2} d T \\
& +C_{2} .
\end{aligned}
$$

As in the DEP array, we can arbitrarily choose up to three points along the real axis of the $T$-plane, and therefore fix $T_{B}=1.2, T_{C}=1$, and $T_{D}=0$, as shown in Fig. 9(b). For $T$ $>T_{E}>T_{B}>T_{C}>T_{D}$, the solution of Eq. (B1) is an elliptic integral [48]:

$$
\begin{aligned}
Z & =C_{3} F\left(\varpi_{t 1}, k_{t 1}\right)+C_{2} \\
& =C_{3} \int_{0}^{\lambda_{t 1}} \frac{d \lambda_{t 1}}{\left(1-\lambda_{t 1}^{2}\right)\left(1-k_{t 1}^{2} \lambda_{t 1}^{2}\right)} d \lambda_{t 1}+C_{2},
\end{aligned}
$$

where $F\left(\varpi_{t 1}, k_{t 1}\right)$ is the elliptical integral of the first kind and $k_{t 1}$ is the modulus of the elliptic function. The expressions for $C_{3}, \varpi_{t 1}, k_{t 1}$, and $\lambda_{t 1}$ are given in Appendix B.

Equation (27) links the $T$-plane with the $Z$-plane. The values of the coefficients $C_{2}$ and $C_{3}$ can be solved by the coordinates of the corresponding points: $B, C, D$, and $E$ (see details in Appendix B)

$$
C_{2}=0, \quad C_{3}=\frac{h}{K\left(k_{t 1}\right)}=\frac{w+g}{K\left(k^{\prime}{ }_{t 1}\right)},
$$

where $K\left(k_{t 1}\right)$ is the complete elliptic integral of the first kind and $k_{t 1}^{\prime}=\sqrt{1-k_{t 1}^{2}}$. Similar to the DEP array case, the expression for $C_{3}$ provides the relationship between the complete elliptical integral and the geometrical parameters of the system:

$$
\frac{K\left(k_{t 1}\right)}{K\left(k^{\prime}{ }_{t 1}\right)}=\frac{h}{w+g} .
$$

The factor of 2 difference compared to the relationship for the DEP array [Eq. (7)] is because the basic cell for twDEP includes two electrodes. Equation (29) can also be evaluated using Hilberg's approximation [49]. The value of the modulus, $k_{t 1}$, is calculated by inputting arbitrary geometrical parameters.

The inverse function of Eq. (27) gives $T$ in terms of $Z$ :

$$
T=\frac{T_{E} T_{B} \mathrm{cn}^{2}\left(\frac{Z}{C_{3}}, k_{t 1}\right)}{T_{B}-T_{E} \operatorname{sn}^{2}\left(\frac{Z}{C_{3}}, k_{t 1}\right)},
$$

where $\operatorname{sn}(\ldots, \ldots)$ and $\operatorname{cn}(\ldots, \ldots)$ are the Jacobian elliptic functions. The coordinate of point $A$ and $F$ in the $T$-plane can 
be obtained from Eq. (30) (see Appendix B).

The SCM method is then used a second time, with the upper half of the $T$-plane transformed into a rectangle in the model plane ( $W$-plane), where the electric field distribution is uniform. The four corner points in the $W$-plane are: $W_{A}$ $=j Y_{W t}, W_{B}=X_{W t}+j Y_{W t}, W_{D}=X_{W t}$ and $W_{F}=0$ where $X_{W t}$ and $Y_{W t}$ are the size of the region along the real and imaginary axes, respectively. The transformation from the $T$-plane to the $W$-plane is given by

$$
\begin{aligned}
W= & D_{1} \int^{T}\left(T-T_{F}\right)^{-1 / 2}\left(T-T_{A}\right)^{-1 / 2}\left(T-T_{B}\right)^{-1 / 2}\left(T-T_{D}\right)^{-1 / 2} d T \\
& +D_{2} .
\end{aligned}
$$

It should be noted that compared to Eq. (26), in this transformation points $A$ and $F$ replace $C$ and $E$ to become two corners of the rectangle. The integral solution of Eq. (31) is

$$
\begin{aligned}
W & =D_{3} F\left(\varpi_{t 2}, k_{t 2}\right)+D_{2} \\
& =D_{3} \int_{0}^{\lambda_{t 2}} \frac{d \lambda_{t 2}}{\left(1-\lambda_{t 2}^{2}\right)\left(1-k_{t 2}^{2} \lambda_{t 2}^{2}\right)} d \lambda_{t 2}+D_{2},
\end{aligned}
$$

with

$$
\begin{gathered}
D_{3}=\frac{2 D_{1}}{\sqrt{\left(T_{A}-T_{D}\right)\left(T_{F}-T_{B}\right)}}, \\
\varpi_{t 2}=\arcsin \left(\sqrt{\frac{\left(T_{A}-T_{D}\right)\left(T-T_{F}\right)}{\left(T_{F}-T_{D}\right)\left(T-T_{A}\right)}}\right), \\
k_{t 2}=\sqrt{\frac{\left(T_{F}-T_{D}\right)\left(T_{A}-T_{B}\right)}{\left(T_{A}-T_{D}\right)\left(T_{F}-T_{B}\right)}}, \quad \lambda_{t 2}=\sin \varpi_{t 2} .
\end{gathered}
$$

Equation (32) gives the transformation from the $T$-plane to $W$-plane. The values of the coefficients $D_{2}$ and $D_{3}$ can be obtained by a similar point-point mapping procedure as presented in Appendix B for the first SCM transformation:

$$
D_{2}=0, \quad D_{3}=\frac{X_{W t}}{K\left(k_{t 2}\right)}=\frac{Y_{W t}}{K\left(k_{t 2}^{\prime}\right)} .
$$

\section{Analytical electric field solution}

Using the relationship between the gradient of the potential in the $Z$ - and $W$-planes [42] and combining Eqs. (26) and (31), the real part of the electric field distribution in the $Z$-plane, $\mathbf{E}_{Z R t}$, for the twDEP array can be derived as

$$
\begin{aligned}
\mathbf{E}_{Z R t} & =-\nabla \phi_{Z R t}=-\nabla \phi_{W R t} \overline{\left(\frac{d W}{d Z}\right)} \\
& =j \frac{V}{h} \frac{K\left(k_{t 1}\right)}{K\left(k^{\prime}{ }_{t 2}\right)}\left[\frac{T_{A}\left(T_{F}-T_{B}\right)\left(T-T_{C}\right)\left(T-T_{E}\right)}{T_{B}\left(T_{E}-T_{C}\right)\left(T-T_{A}\right)\left(T-T_{F}\right)}\right]^{1 / 2},
\end{aligned}
$$

where $\phi_{Z R t}$ and $\phi_{W R t}$ are the real part of the potential in the $Z$ - and $W$-plane, respectively.

Equation (34) is the analytical solution for the real part of the electric field distribution in the basic cell for the twDEP
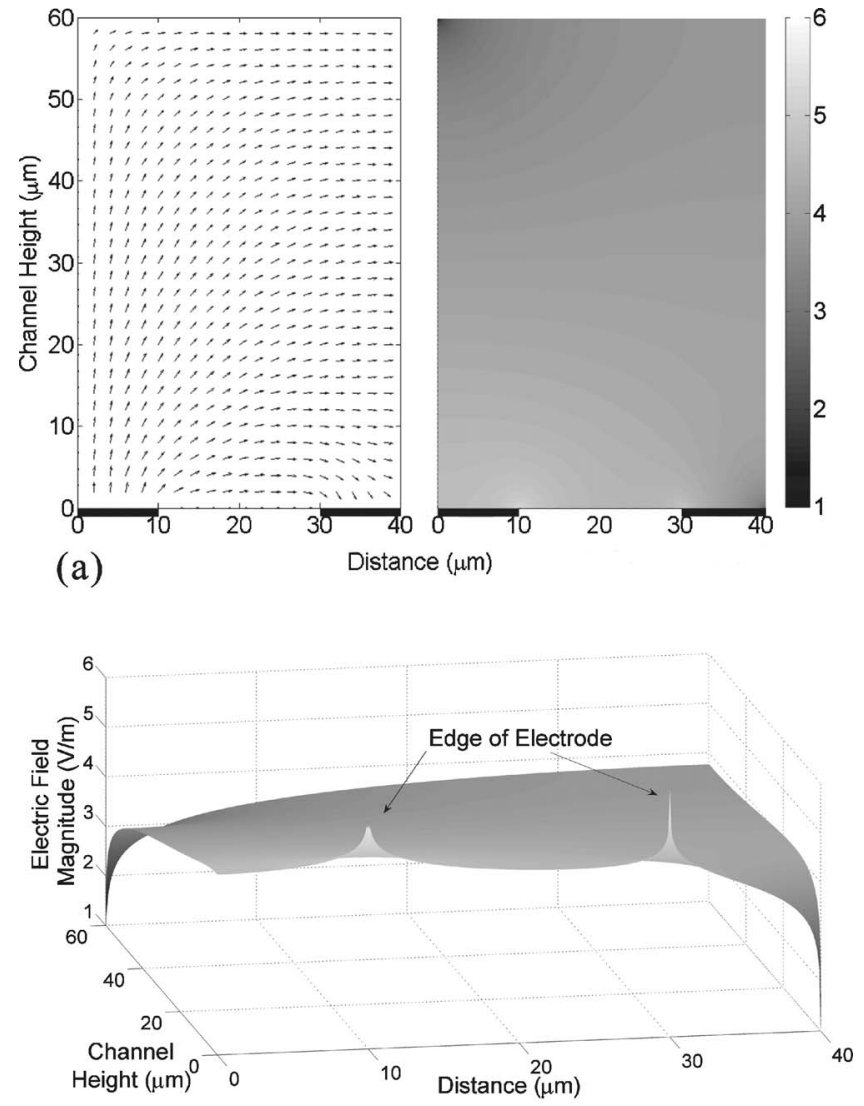

(b)

FIG. 10. Solutions of the real part of the electric field in the basic cell of the twDEP array. The positions of the electrodes are drawn on the figures. (a) Electric field vectors and magnitude $(\mathrm{V} / \mathrm{m}$, $\left.\log _{10}\right)$. (c) 3D surface plot of electric field magnitude $\left(\mathrm{V} / \mathrm{m}, \log _{10}\right)$.

array. The expressions for $T_{A}, T_{E}$, and $T_{F}$ are given in Appendix $\mathrm{B}$ and $T_{B}=1.2, T_{C}=1$. Similar features of the field distribution can be seen as for the DEP array case. The electric field magnitude goes to zero at points $C$ and $E$ (when $T=T_{C}$ and $T=T_{E}$ ) and infinity at points $A$ and $F$ (when $T$ $=T_{A}$ and $T=T_{F}$ ), which represent the electrode edges.

Equation (34) is then used to calculate the electric field terms in the expressions for the dielectrophoretic force, the traveling wave dielectrophoretic force and electrorotational torque. The calculations were performed in MATLAB ${ }^{\mathrm{TM}}$. For the twDEP array case, we only consider a design of array which includes the lid of the channel. A simpler solution with the channel height going to infinity can be derived, as in the DEP array case, but will not be presented here. The width of the electrode and the gap separation was set to $20 \mu \mathrm{m}$ and the height of the channel to $60 \mu \mathrm{m}$. The voltage applied to the electrode is 1 volt.

\section{Electric field distribution}

Figure 10(a) shows the direction and the magnitude of the real part of the electric field, $\mathbf{E}_{Z R t}$, for the basic cell. Figure $10(\mathrm{~b})$ is a $3 \mathrm{D}$ surface plot of the magnitude of the real part of the electric field, which goes to a maximum at the edge of 


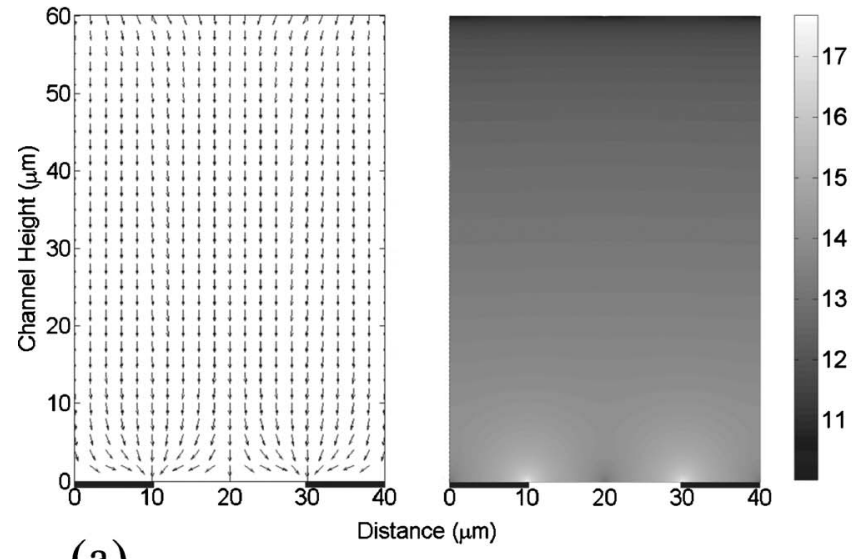

(a)

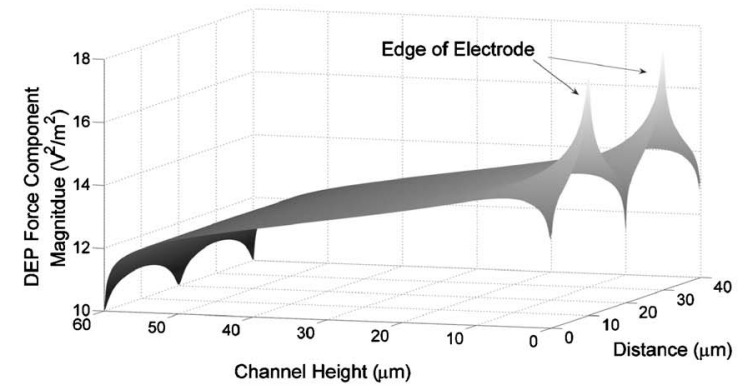

(b)

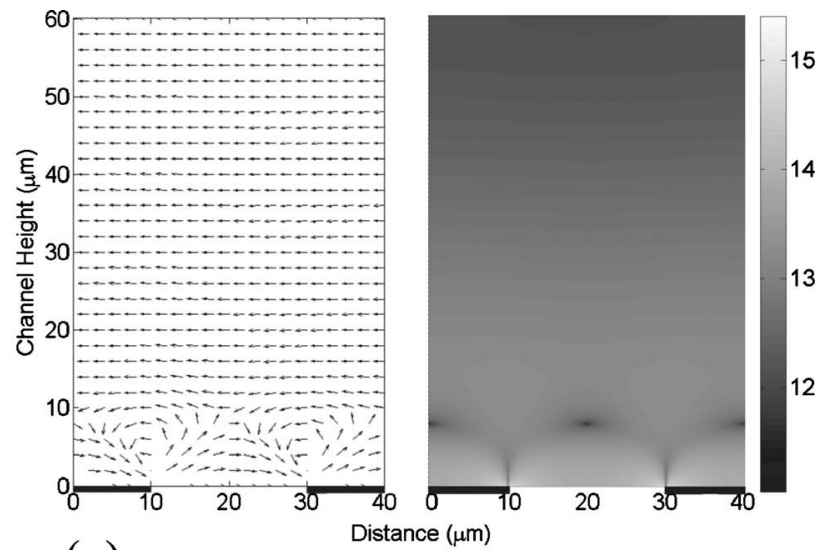

(c)

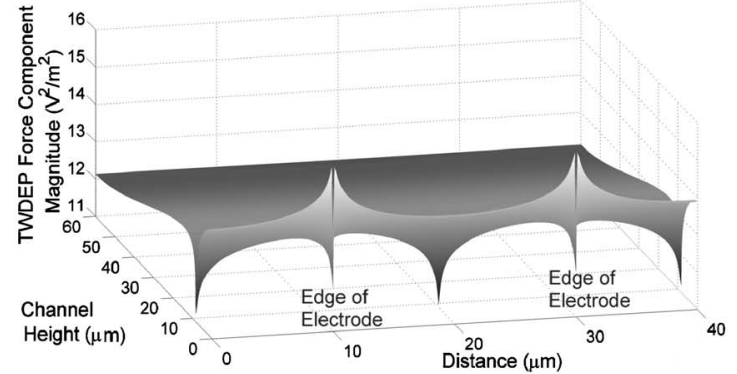

(d)

FIG. 11. Solutions of the DEP force component $\left(\nabla\left|\mathbf{E}_{Z t}\right|^{2}\right)$ and twDEP force component $\left[\nabla \times\left(\mathbf{E}_{Z R t} \times \mathbf{E}_{Z I t}\right)\right]$ in the basic cell of the twDEP array. (a) DEP force component, vectors, and magnitude $\left(\log _{10}\right)$. (b) 3D surface plot of the magnitude of the DEP force component $\left(\log _{10}\right)$. (c) twDEP force component, vectors, and magnitude $\left(\log _{10}\right)$. (d) 3D surface plot of the magnitude of the twDEP force component.

the electrodes (points $A$ and $F$ ) and decreases away from the electrodes. The zero field points (point $C$ and $E$ ) can clearly be seen in the figure. The solution of $\mathbf{E}_{Z I t}$, required to correctly determine the forces and torques in the array, is obtained by mirroring the solution for $\mathbf{E}_{Z R t}$ about the vertical line through the horizontal position $20 \mu \mathrm{m}$.

\section{E. DEP and twDEP force}

Figure 11(a) shows the direction and the magnitude of $\nabla\left|\mathbf{E}_{Z t}\right|^{2}$ in the basic cell. The behavior is similar to the solution presented for the DEP case. Above a certain height, the directions of the DEP force at every position points straight toward the electrode plane, with an exponentially decreasing magnitude. However, as in the DEP array with complete boundary conditions, the channel lid disturbs this behavior and the DEP force is horizontal at the lid. Figure 11(b) shows the 3D surface plot of the magnitude of $\boldsymbol{\nabla}\left|\mathbf{E}_{Z t}\right|^{2}$, clearly showing the maxima at the edges of the electrode and the exponential decreases away from the electrodes. The minima in the center of the electrodes and the gap can also be seen, as well as corresponding minima at the insulating lid.

Figure 11(c) shows the direction and the magnitude of the twDEP force component $\left[\nabla \times\left(\mathbf{E}_{Z R t} \times \mathbf{E}_{Z I t}\right)\right]$. Far from the electrodes (above $10 \mu \mathrm{m}$ ), the vectors point in the negative $x$-direction (left). Approaching the bottom, the vectors point in the opposite direction. In the region directly above the edges of the electrode, the vectors show a circular pattern, an observation identical to previous numerical simulations [30]. From the plot of the magnitude, it is observed that the distribution of the twDEP force component is more complicated than the DEP force. There are three twDEP force minima in the near field region (approximately at a height of $10 \mu \mathrm{m}$ ). Along the surface of the electrode, the magnitude of twDEP force increases towards the edge of the electrode. However, it does not go to a maximum above the edge of the electrodes. Instead, it rapidly drops to a minimum over the electrode edge because the vectors are recirculating in this region. This can be observed more clearly in the 3D surface plot, as shown in Fig. 11(d).

\section{F. ROT torque}

The field term in electrorotation, which we will refer to as the ROT torque component, is $\mathbf{E}_{Z R t} \times \mathbf{E}_{Z I t}$. Figures 12(a) and 12(b) show the magnitude of the ROT torque in $2 \mathrm{D}$ and $3 \mathrm{D}$ plots, respectively. The direction of the torque is in the third dimension (i.e., out of the page). The magnitude of the torque goes to a maximum at the edge of the electrode. Beyond the near field region, the magnitude of torque decreases with distance from the electrode. 

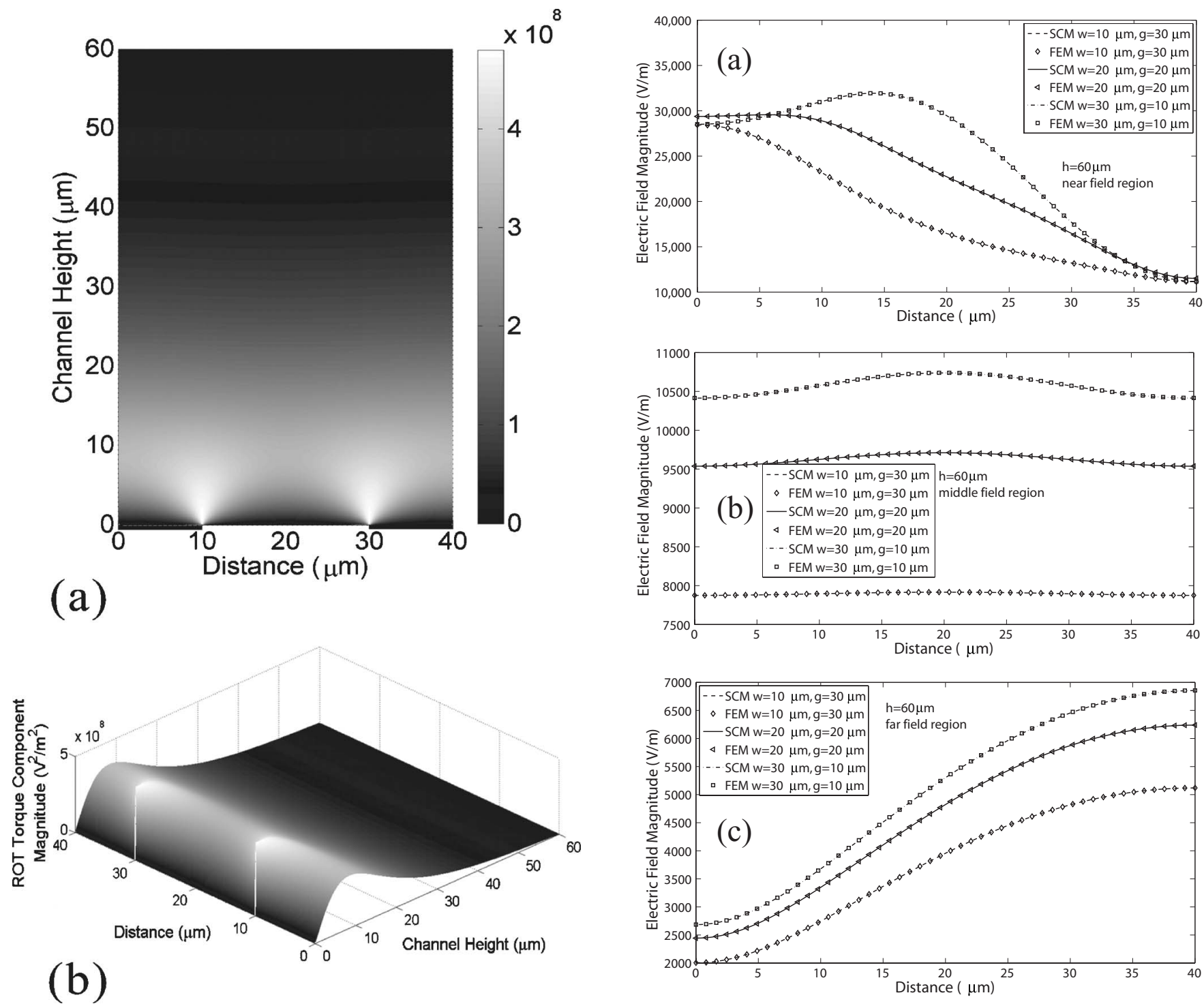

FIG. 12. Solutions of the ROT torque component $\left(\mathbf{E}_{Z R t} \times \mathbf{E}_{Z I t}\right)$ in the basic cell of the twDEP array. (a) Magnitude of the ROT torque component magnitude in $2 \mathrm{D}$. (b) $3 \mathrm{D}$ surface plot of the magnitude of the ROT torque.

\section{G. Comparison with FEM simulations}

The analytical solution for the real part of the electric field was compared with numerical simulations, performed using the finite element method (FEM) in COMSOL MULTIPHYSICS ${ }^{\mathrm{TM}}$. The comparisons are performed by varying the ratio of the electrode width and gap, with the channel height set at $60 \mu \mathrm{m}$. The sum of the electrode width and gap is fixed at $40 \mu \mathrm{m}$, with the ratio of electrode width to gap distance varied from 1:3 $(w=10 \mu \mathrm{m}, g=30 \mu \mathrm{m}), 1: 1(w$ $=20 \mu \mathrm{m}, g=20 \mu \mathrm{m})$, and 3:1 ( $w=30 \mu \mathrm{m}, g=10 \mu \mathrm{m})$. Comparisons of the electric field magnitude are performed at the height of $10 \mu \mathrm{m}$ (near field region), $30 \mu \mathrm{m}$ (middle field region), and $50 \mu \mathrm{m}$ (far field region), respectively. Figures 13(a)-13(c) show excellent agreements between the SCM solutions and FEM solutions for the three ratios and the three regions, respectively. This plot validates the analytical solu-

FIG. 13. Plot showing excellent agreements between the electric field distribution calculated with the SCM method and FEM simulations for different geometries. (a) Near field region, (b) midfield region, and (c) far field region.

tions for the DEP, twDEP force, and ROT torque in the traveling wave interdigitated electrode arrays.

\section{CONCLUSION}

In this paper, the analytical solutions for the electric field distributions above interdigitated electrode arrays used for dielectrophoresis and traveling wave dielectrophoresis have been derived using the Schwarz-Christoffel mapping method. The analytical solutions for electric field for the DEP and twDEP arrays are related to the geometrical constant of the device: electrode length, gap distance and the channel height. The field solutions are then used to determine the DEP force, twDEP force, and ROT torque in the corresponding system. The analytical electric field solutions in both cases are validated by comparison with the finite ele- 
ment method, for different geometries, showing excellent agreement. This represents the first complete analytical electric field solutions for these interdigitated electrode arrays without any approximation to the boundary conditions. These solutions are straightforward and easy to use and will provide a significant improvement in field modeling and aid in modeling for the translational movement of particles in real systems.

\section{ACKNOWLEDGMENTS}

The work is partly supported by the funding from the Life Science Initiative of the University of Southampton. The authors would like to thank Antonio Ramos for useful discussions.

\section{APPENDIX A: SCHWARZ-CHRISTOFFEL MAPPING METHOD}

In this appendix, we give the general mathematical description of Schwarz-Christoffel mapping (SCM) method. Details can be found in [31].

Let $\wp$ be an $m$-sided polygon in the $Z$-plane with vertices $Z_{1}, Z_{2} \ldots, Z_{m}$ and interior angles $\theta_{1}, \theta_{2}, \ldots, \theta_{m}$, respectively. Along the real axis of the $T$-plane, $T_{1}, T_{2}, \ldots, T_{m}$ are the corresponding mapping points to $Z_{1}, Z_{2}, \ldots, Z_{m}$ in the $Z$-plane. The SCM integral, which maps the upper half of the $T$-plane into the interior of $\wp$ in the $Z$-plane, is given by

$$
Z=C_{1} \int_{T_{0}}^{T} \prod_{r=1}^{m}\left(T-T_{r}\right)^{\left(\theta_{r} / \pi-1\right)} d T+C_{2}
$$

where $C_{1}$ and $C_{2}$ are integral coefficients. $C_{1}$ establishes the scale and orientation of the polygon in the Z-plane and $C_{2}$ gives its position. These coefficients can be determined from the positions of the corresponding points in the $Z$ - and $T$-planes. The mapping system has three degrees of freedom which means up to three points can be chosen arbitrarily along the real axis of the $T$-plane. The point $T_{0}$ is the reference point, which is usually chosen to be the origin.

\section{APPENDIX B: MAPPING CALCULATIONS IN THE ANALYSIS OF DEP AND twDEP ARRAYS}

In this appendix, the detailed calculations to determine the coordinates of the mapping points in the DEP and twDEP arrays are presented.

\section{The DEP electrode array}

The expressions of the variable $C_{3}, \varpi_{d 1}, k_{d 1}$, and $\lambda_{d 1}$ in Eq. (5) are

$$
C_{3}=\frac{2 C_{1}}{\sqrt{\left(T_{B}-T_{D}\right)\left(T_{E}-T_{C}\right)}},
$$

$$
\begin{gathered}
\varpi_{d 1}=\arcsin \left(\sqrt{\frac{\left(T_{B}-T_{D}\right)\left(T-T_{E}\right)}{\left(T_{E}-T_{D}\right)\left(T-T_{B}\right)}}\right), \\
k_{d 1}=\sqrt{\frac{\left(T_{E}-T_{D}\right)\left(T_{B}-T_{C}\right)}{\left(T_{B}-T_{D}\right)\left(T_{E}-T_{C}\right)}}, \\
\lambda_{d 1}=\sin \varpi_{d 1} .
\end{gathered}
$$

The values of the coefficients $C_{2}$ and $C_{3}$ in Eq. (5) are solved by mapping the coordinates of the corresponding points in the $Z$ - and $T$-planes.

Taking point $E, T=T_{E}$, giving $\lambda_{d 1}=0$ and Eq. (5) becomes

$$
Z_{E}=C_{2}=0 \text {. }
$$

Taking point $B, T=T_{B}$, giving $\lambda_{d 1}=\infty$ and Eq. (5) becomes

$$
Z_{B}=C_{3} \int_{0}^{\infty} \frac{d \lambda_{d 1}}{\left(1-\lambda_{d 1}^{2}\right)\left(1-k_{d 1}^{2} \lambda_{d 1}^{2}\right)} d \lambda_{d 1}=C_{3} j K\left(k_{d 1}^{\prime}\right)=j \frac{w+g}{2} .
$$

Taking point $D, T=T_{D}$, giving $\lambda_{d 1}=1$ and Eq. (5) becomes

$$
Z_{D}=C_{3} \int_{0}^{1} \frac{d \lambda_{d 1}}{\left(1-\lambda_{d 1}^{2}\right)\left(1-k_{d 1}^{2} \lambda_{d 1}^{2}\right)} d \lambda_{d 1}=C_{3} K\left(k_{d 1}\right)=h .
$$

Taking point $C, T=T_{C}$, giving $\lambda_{d 1}=1 / k_{d 1}$ and Eq. (5) becomes

$$
\begin{aligned}
Z_{C} & =C_{3} \int_{0}^{1 / k_{d 1}} \frac{d \lambda_{d 1}}{\left(1-\lambda_{d 1}^{2}\right)\left(1-k_{d 1}^{2} \lambda_{d 1}^{2}\right)} d \lambda_{d 1} \\
& =C_{3}\left[K\left(k_{d 1}\right)+j K\left(k_{d 1}^{\prime}\right)\right]=h+j \frac{w+g}{2} .
\end{aligned}
$$

Combining Eqs. (B3)-(B5) gives

$$
C_{3}=\frac{h}{K\left(k_{d 1}\right)}=\frac{w+g}{2 K\left(k_{d 1}^{\prime}\right)},
$$

where $K\left(k_{d 1}\right)$ is the complete elliptic integral of the first kind and $k_{d 1}^{\prime}=\sqrt{1-k_{d 1}^{2}}$.

Since the value of $k_{d 1}$ can be derived from Eq. (7), according to Eq. (B1) the value of $T_{E}$ is given by

$$
T_{E}=\frac{T_{C}\left(T_{B}-T_{D}\right) k_{d 1}^{2}-T_{D}\left(T_{B}-T_{C}\right)}{\left(T_{B}-T_{D}\right) k_{d 1}^{2}-\left(T_{B}-T_{C}\right)} .
$$

Then the coordinate of point $A$ in the $T$-plane can be obtained from Eq. (8):

$$
T_{A}=\frac{T_{E} T_{B} \mathrm{cn}^{2}\left(j \frac{g K\left(k_{d 1}^{\prime}\right)}{w+g}, k_{d 1}\right)}{T_{B}-T_{E} \operatorname{sn}^{2}\left(j \frac{g K\left(k_{d 1}^{\prime}\right)}{w+g}, k_{d 1}\right)} .
$$

The expressions of the variable $D_{3}, \varpi_{d 2}, k_{d 2}$ and $\lambda_{d 2}$ in Eq. (10) are

$$
D_{3}=\frac{2 D_{1}}{\sqrt{\left(T_{A}-T_{D}\right)\left(T_{E}-T_{B}\right)}}
$$




$$
\begin{gathered}
\varpi_{d 2}=\arcsin \left(\sqrt{\frac{\left(T_{A}-T_{D}\right)\left(T-T_{E}\right)}{\left(T_{E}-T_{D}\right)\left(T-T_{A}\right)}}\right), \\
k_{d 2}=\sqrt{\frac{\left(T_{E}-T_{D}\right)\left(T_{A}-T_{B}\right)}{\left(T_{A}-T_{D}\right)\left(T_{E}-T_{B}\right)}}, \\
\lambda_{d 2}=\sin \varpi_{d 2} .
\end{gathered}
$$

The formulas for separating the real and imaginary parts of the Jacobian elliptic functions are

$$
\begin{aligned}
\operatorname{sn}(u+j v, k)= & \frac{\operatorname{sn}(u, k) \operatorname{dn}\left(v, k^{\prime}\right)}{1-\operatorname{sn}^{2}\left(v, k^{\prime}\right) \operatorname{dn}^{2}(u, k)} \\
& +j \frac{\operatorname{cn}(u, k) \operatorname{dn}(u, k) \operatorname{sn}\left(v, k^{\prime}\right) \operatorname{cn}\left(v, k^{\prime}\right)}{1-\operatorname{sn}^{2}\left(v, k^{\prime}\right) \operatorname{dn}^{2}(u, k)},
\end{aligned}
$$

$$
\begin{aligned}
\operatorname{cn}(u+j v, k)= & \frac{\operatorname{cn}(u, k) \operatorname{cn}\left(v, k^{\prime}\right)}{1-\operatorname{sn}^{2}\left(v, k^{\prime}\right) \operatorname{dn}^{2}(u, k)} \\
& +j \frac{\operatorname{sn}(u, k) \operatorname{dn}(u, k) \operatorname{sn}\left(v, k^{\prime}\right) \operatorname{dn}\left(v, k^{\prime}\right)}{1-\operatorname{sn}^{2}\left(v, k^{\prime}\right) \operatorname{dn}^{2}(u, k)},
\end{aligned}
$$

where $\operatorname{sn}(\ldots, \ldots), \mathrm{cn}(\ldots, \ldots)$, and $\operatorname{dn}(\ldots, \ldots)$ are the Jacobian elliptic functions.

\section{2. twDEP electrode array}

The expressions for the variable $C_{3}, \varpi_{t 1}, k_{t 1}$ and $\lambda_{t 1}$ in Eq. (27) are

$$
\begin{gathered}
C_{3}=\frac{2 C_{1}}{\sqrt{\left(T_{B}-T_{D}\right)\left(T_{E}-T_{C}\right)}}, \\
\varpi_{t 1}=\arcsin \left(\sqrt{\frac{\left(T_{B}-T_{D}\right)\left(T-T_{E}\right)}{\left(T_{E}-T_{D}\right)\left(T-T_{B}\right)}}\right), \\
k_{t 1}=\sqrt{\frac{\left(T_{E}-T_{D}\right)\left(T_{B}-T_{C}\right)}{\left(T_{B}-T_{D}\right)\left(T_{E}-T_{C}\right)}}, \\
\lambda_{t 1}=\sin \varpi_{t 1} .
\end{gathered}
$$

Similarly to the DEP case, the values of the coefficients $C_{2}$ and $C_{3}$ in Eq. (27) are solved by mapping the coordinates of the corresponding points in the $Z$ - and $T$-planes for the twDEP array.

Taking point $E, T=T_{E}$, giving $\lambda_{t 1}=0$ and Eq. (27) becomes

$$
Z_{E}=C_{2}=0 \text {. }
$$

Taking point $B, T=T_{B}$, giving $\lambda_{t 1}=\infty$ and Eq. (27) becomes $Z_{B}=C_{3} \int_{0}^{\infty} \frac{d \lambda_{t 1}}{\left(1-\lambda_{t 1}^{2}\right)\left(1-k_{t 1}^{2} \lambda_{t 1}^{2}\right)} d \lambda_{t 1}=C_{3} j K\left(k^{\prime}{ }_{t 1}\right)=j(w+g)$.

Taking point $D, T=T_{D}$, giving $\lambda_{t 1}=1$ and Eq. (27) becomes

$$
Z_{D}=C_{3} \int_{0}^{1} \frac{d \lambda_{t 1}}{\left(1-\lambda_{t 1}^{2}\right)\left(1-k_{t 1}^{2} \lambda_{t 1}^{2}\right)} d \lambda_{t 1}=C_{3} K\left(k_{t 1}\right)=h
$$

Taking point $C, T=T_{C}$, giving $\lambda_{t 1}=1 / k_{t 1}$ and Eq. (27) becomes

$$
\begin{aligned}
Z_{C} & =C_{3} \int_{0}^{1 / k_{t 1}} \frac{d \lambda_{t 1}}{\left(1-\lambda_{t 1}^{2}\right)\left(1-k_{t 1}^{2} \lambda_{t 1}^{2}\right)} d \lambda_{t 1} \\
& =C_{3}\left[K\left(k_{t 1}\right)+j K\left(k^{\prime}{ }_{t 1}\right)\right]=h+j(w+g) .
\end{aligned}
$$

Combining Eqs. (B13)-(B15) gives

$$
C_{3}=\frac{h}{K\left(k_{t 1}\right)}=\frac{w+g}{K\left(k^{\prime}{ }_{t 1}\right)},
$$

where $K\left(k_{t 1}\right)$ is the complete elliptic integral of the first kind and $k_{t 1}^{\prime}=\sqrt{1-k_{t 1}^{2}}$.

Also from Eq. (B11c), the expression for $T_{E}$ is

$$
T_{E}=\frac{T_{C}\left(T_{B}-T_{D}\right) k_{t 1}^{2}-T_{D}\left(T_{B}-T_{C}\right)}{\left(T_{B}-T_{D}\right) k_{t 1}^{2}-\left(T_{B}-T_{C}\right)}
$$

The coordinate of point $A$ and $F$ in the $T$-plane can be obtained from Eq. (30):

$$
\begin{gathered}
T_{A}=\frac{T_{E} T_{B} \mathrm{cn}^{2}\left(j \frac{(w+2 g) K\left(k_{t 1}\right)}{2 h}, k_{t 1}\right)}{T_{B}-T_{E} \operatorname{sn}^{2}\left(j \frac{(w+2 g) K\left(k_{t 1}\right)}{2 h}, k_{t 1}\right)}, \\
T_{F}=\frac{T_{E} T_{B} \mathrm{cn}^{2}\left(j \frac{w K\left(k_{t 1}\right)}{2 h}, k_{t 1}\right)}{T_{B}-T_{E} \operatorname{sn}^{2}\left(j \frac{w K\left(k_{t 1}\right)}{2 h}, k_{t 1}\right)} .
\end{gathered}
$$

[1] H. Morgan and N. G. Green, AC Electrokinetics: Colloids and Nanoparticles (Research Studies Press, Ltd. Baldock, Hertfordshire, England, 2003).

[2] Y. Huang, X. B. Wang, J. A. Tame, and R. Pethig, J. Phys. D 26, 1528 (1993).

[3] P. R. C. Gascoyne, X. B. Wang, Y. Huang, and F. F. Becker,
IEEE Trans. Ind. Appl. 33, 670 (1997).

[4] M. P. Hughes and H. Morgan, Biotechnol. Prog. 15, 245 (1999).

[5] L. Cui, D. Holmes, and H. Morgan, Electrophoresis 22, 3893 (2001).

[6] E. B. Cummings and A. K. Singh, Anal. Chem. 75, 4724 
(2003).

[7] B. H. Lapizco-Encinas, B. A. Simmons, E. B. Cummings, and Y. Fintschenko, Anal. Chem. 76, 1571 (2004).

[8] J. Voldman, Annu. Rev. Biomed. Eng. 8, 425 (2006).

[9] H. A. Pohl, Dielectrophoresis (Cambridge University Press, New York, 1978).

[10] B. H. Lapizco-Encinas, R. V. Davalos, B. A. Simmons, E. B. Cummings, and Y. Fintschenko, J. Microbiol. Methods 62, 317 (2005).

[11] J. P. Huang, K. W. Yu, and G. Q. Gu, Phys. Lett. A 300, 385 (2002).

[12] G. Liao, I. I. Smalyukh, J. R. Kelly, O. D. Lavrentovich, and A. Jakli, Phys. Rev. E 72, 031704 (2005).

[13] H. Morgan, N. G. Green, M. P. Hughes, W. Monaghan, and T. C. Tan, J. Micromech. Microeng. 7, 65 (1997).

[14] L. Cui and H. Morgan, J. Micromech. Microeng. 10, 72 (2000).

[15] H. Kawamoto, K. Seki, and N. Kuromiya, J. Phys. D 39, 1249 (2006)

[16] N. G. Green and H. Morgan, J. Phys. Chem. B 103, 41 (1999).

[17] I. Ermolina and H. Morgan, J. Colloid Interface Sci. 285, 419 (2005).

[18] S. Archer, H. Morgan, and J. F. Rixon, J. Biol. Phys. 76, 2833 (1999).

[19] C. Dalton, A. D. Goater, J. P. H. Burt, and H. V. Smith, J. Appl. Microbiol. 96, 24 (2004).

[20] X.-B. Wang, R. Pethig, and T. B. Jones, J. Phys. D 25, 905 (1992).

[21] X.-B. Wang, Y. Huang, R. Holzel, J. P. H. Burt, and R. Pethig, J. Phys. D 26, 312 (1993).

[22] S. Masuda, M. Washizu, and I. Kawabata, IEEE Trans. Ind. Appl. 24, 214 (1988).

[23] X. Wang, X.-B. Wang, F. F. Becker, and P. R. C. Gascoyne, J. Phys. D 29, 1649 (1996).

[24] M. Garcia and D. S. Clague, J. Phys. D 33, 1747 (2000).

[25] E. Liang, R. L. Smith, and D. S. Clague, Phys. Rev. E 70, 066617 (2004).

[26] D. S. Clague and E. K. Wheeler, Phys. Rev. E 64, 026605 (2001).

[27] H. Morgan, A. G. Izquierdo, D. Bakewell, N. G. Green, and A. Ramos, J. Phys. D 34, 1553 (2001).

[28] D. E. Chuang, S. Loire, and I. Mezic, J. Phys. D 36, 3073 (2003).

[29] X.-B. Wang, Y. Huang, J. P. H. Burt, G. H. Markx, and R.
Pethig, J. Phys. D 26, 1278 (1993).

[30] N. G. Green, A. Ramos, and H. Morgan, J. Electrost. 56, 235 (2002).

[31] R. Schinzinger and P. A. A. Laurra, Conformal Mapping: Methods and Applications (Dover Publications, Inc., Mineola, NY, 2003).

[32] C. K. Ikoc and P. F. Ordung, IEEE Trans. Comput.-Aided Des. 8, 1025 (1989).

[33] A. Balakrishnan, W. T. Joines, and T. G. Wilson, IEEE Trans. Power Electron. 12, 654 (1997).

[34] E. Costamagna and A. Fanni, 8th Electrotechnical Conf. 3, 1393 (1996).

[35] G. Ghione, M. Goano, and M. Pirola, IEEE MTT-S Int. Microwave Symp. Dig. 3, 1311 (1999).

[36] M. Goano, F. Bertazzi, P. Caravelli, G. Ghione, and T. A. Driscoll, IEEE Trans. Microwave Theory Tech. 49, 1573 (2001).

[37] V. Teppati, M. Goano, and A. Ferrero, IEEE Trans. Microwave Theory Tech. 50, 2339 (2002).

[38] A. A. Bilotti, IEEE Trans. Electron Devices 21, 217 (1974).

[39] M. Chung, P.-S. Jung, and R. H. Rangel, Int. J. Heat Mass Transfer 43, 3771 (1999).

[40] J. M. Chuang, Int. J. Numer. Methods Fluids 32, 745 (2000).

[41] P. Bruschi, A. Nannini, F. Pieri, G. Raffa, B. Vigna, and S. Zerbini, Sens. Actuators, A 113, 106 (2004).

[42] M. Markovic, M. Jufer, and Y. Perriard, IEEE Trans. Magn. 40, 1858 (2004).

[43] S. Gevorgian, T. Martinsson, P. L. J. Linnér, and E. L. Kollberg, IEEE Trans. Microwave Theory Tech. 44, 896 (1996).

[44] E. Carlsson and S. Gevorgian, IEEE Trans. Microwave Theory Tech. 47, 1544 (1999).

[45] S. Gevorgian, H. Berg, H. Jacobsson, and T. Lewin, IEEE Microw. Mag. 4, 60 (2003).

[46] S. Gevorgian, H. Berg, H. Jacobsson, and T. Lewin, IEEE Microw. Mag. 4, 59 (2003).

[47] N. G. Green, A. Ramos, A. González, A. Castellanos, and H. Morgan, J. Electrost. 53, 71 (2001).

[48] I. S. Gradshteyn and I. M. Ryzhik, Table of Integrals, Series and Products, 5th ed. (Academic Press, San Diego, New York, 1994).

[49] W. Hilberg, IEEE Trans. Microwave Theory Tech. 17, 259 (1969).

[50] P. F. Byrd and M. D. Friedman, Handbook of Elliptic Integrals for Engineers and Physicists (Springer-Verlag, Berlin, 1954). 